University of Nebraska - Lincoln

DigitalCommons@University of Nebraska - Lincoln

3-2009

\title{
The Dynamic Nature of Knowledge: Insights from a Dynamic Field Model of Children's Novel Noun Generalization
}

Larissa K. Samuelson

University of lowa, larissa-samuelson@uiowa.edu

Anne R. Schutte

University of Nebraska-Lincoln, aschutte2@unl.edu

Jessica S. Horst

University of Sussex

Follow this and additional works at: https://digitalcommons.unl.edu/psychfacpub

Part of the Psychiatry and Psychology Commons

Samuelson, Larissa K.; Schutte, Anne R.; and Horst, Jessica S., "The Dynamic Nature of Knowledge: Insights from a Dynamic Field Model of Children's Novel Noun Generalization" (2009). Faculty Publications, Department of Psychology. 498.

https://digitalcommons.unl.edu/psychfacpub/498

This Article is brought to you for free and open access by the Psychology, Department of at DigitalCommons@University of Nebraska - Lincoln. It has been accepted for inclusion in Faculty Publications, Department of Psychology by an authorized administrator of DigitalCommons@University of Nebraska - Lincoln. 


\title{
The Dynamic Nature of Knowledge: Insights from a Dynamic Field Model of Children's Novel Noun Generalization
}

\author{
Larissa K. Samuelson \\ Department of Psychology and Iowa Center for Developmental and Learning Sciences, \\ University of Iowa, E11 Seashore Hall, Iowa City, IA 52242; \\ Corresponding author - tel 319 335-2211, fax 319 335-0191, email larissa-samuelson@uiowa.edu \\ Anne R. Schutte \\ Department of Psychology, University of Nebraska-Lincoln \\ Jessica S. Horst \\ Department of Psychology, University of Sussex
}

\begin{abstract}
This paper examines the tie between knowledge and behavior in a noun generalization context. An experiment directly comparing noun generalizations of children at the same point in development in forced-choice and yes/no tasks reveals task-specific differences in the way children's knowledge of nominal categories is brought to bear in a moment. To understand the cognitive system that produced these differences, the real-time decision processes in these tasks were instantiated in a dynamic field model. The model captures both qualitative and quantitative differences in performance across tasks and reveals constraints on the nature of children's accumulated knowledge. Additional simulations of developmental change in the yes/no task between 2 and 4 years of age illustrate how changes in children's representations translate into developmental changes in behavior. Together, the empirical data and model demonstrate the dynamic nature of knowledge and are consistent with the perspective that knowledge cannot be separated from the task-specific processes that create behavior in the moment.
\end{abstract}

Keywords: word learning, developmental processes, competence/performance, computational modeling

\section{Introduction}

There are numerous examples in the cognitive development literature where children's abilities appear to shift across ages and tasks in seemingly paradoxical ways. For example, studies with young infants suggest that 3and 4-month-old children can use knowledge of physical laws of continuity and solidity to determine where a ball rolled down a ramp should stop (Spelke, Breinlinger, Macomber, \& Jacobson, 1992). Yet, when tested in similar paradigms, 2- and 3-year-old children do not seem to have this same knowledge (Berthier, DeBlois, Poirier, Novak, \& Clifton, 2000). Similarly, research suggests that 6- to 8-monthold infants can detect the numerical equivalence between sets of auditory and visual stimuli (Starkey, Spelke, \& Gelman, 1990); yet, 3-year-old children fail at a similar task (Mix, Huttenlocher, \& Levine, 1996). Such examples leave the field in a difficult position: how are we to know when a child possesses some bit of knowledge?

This question comes from a view of cognition that separates knowledge from process. By this view, knowledge resides in the head of the child, waiting to be accessed by the appropriate stimuli or task. Thus, developmentalists design tasks to tap into children's knowledge; if children perform competently they are said to have the requisite knowledge, if children fail they are said to lack the knowledge. One problem with this view is that it pits children's competence (i.e., knowledge) against their performance (i.e., behavior). Consequently, when children fail at a task it can always be claimed that they have the knowledge, but that the task did not effectively elicit that knowledge (see Sophian, 1997, and commentaries for discussion). A second problem with this view is that it leaves unexplained what is critical for acting in the world and for the unfolding of developmental process-how knowledge is created and how it is brought to bear in a task. 
An alternative and growing view is that knowledge is not separate from the processes that create behavior in a task. That is, knowledge is not a unitary thing that can be had, rather, it is distributed in and across many processes (e.g. Barsalou, 1999; Plumert, 2008; Port and VanGelder, 1995; Samuelson and Smith, 2000b; Skarda and Freeman, 1987; Spencer and Schöner, 2003; Spivey and Dale, 2006; Thelen and Smith, 1994). By this view, one cannot ask about knowledge independent of the task that brings that knowledge to bear in a moment in time. Rather, the question to be asked is how the specifics of the task cohere with the child's prior history of perceiving, thinking, and acting to create behavior in the moment.

Direct support for this view in developmental science comes from work by Esther Thelen, Linda Smith, and their colleagues showing how infants' performance in the classic Piagetian A-not-B task is influenced by a host of intrinsic and extrinsic factors (Clearfield et al., 2006; Diedrich et al., 2001; Diedrich et al., 2000; Smith et al., 1999; Thelen et al., 2001; Thelen and Smith, 1997). Although such studies have contributed foundational support for the idea that knowledge is bound to behavior in a particular task, the implications of this work for cognition more generally have been called into question given that the A-not-B phenomenon is largely grounded in sensorimotor activity (Freeman, 2001; Glenberg et al., 2001; Markman, 2001; Munakata and McClelland, 2003; but see Spencer \& Schöner, 2003). An important question, then, is whether this view has implications for higher-order cognition.

This question is well illustrated by a current debate in the word learning literature (see Samuelson \& Bloom, 2008). In a typical experimental procedure, a young child shown a novel solid, rigid object and told a novel name (e.g., "this is a dax") will most likely say that only other objects that share the same shape as the exemplar can be called by the same name (Imai et al., 1994; Landau et al., 1988; Samuelson and Smith, 1999). Young children are thus said to show a "shape bias" when generalizing novel names for solid objects. Importantly, however, children do not always generalize novel names by shape similarity. Rather, attention to shape and other object dimensions has been shown to be context, stimulus, and language specific, and thus exquisitely tuned to the language being learned (see Smith and Samuelson, 2006; Yoshida and Smith, 2003). Furthermore, recent studies suggest that children who learn to attend to shape when naming novel objects subsequently show accelerated vocabulary development (Samuelson, 2002; Smith et al., 2002) and that development of a shape bias is related to the development of the early noun vocabulary (Gershkoff-Stowe and Smith, 2004; Samuelson and Smith, 1999).

Nevertheless, there has been sharp debate regarding the origin of the shape bias (Booth and Waxman, 2002; Diesendruck and Bloom, 2003; Smith et al., 2003). At its core, this debate is about the foundational nature of cognition and whether knowledge/competence can be separated from performance (Colunga and Smith, 2008; Samuelson and Horst, 2008; Smith and Samuelson, 2006). Booth, Waxman, and Huang (2005), and Bloom and colleagues (Bloom, 2000; Bloom and Markson, 1998; Diesendruck and Bloom, 2003) argue that children's biased attention to shape reflects their conceptual understanding that shape is an important indicator of object kind. These arguments stem from a traditional view of cognition that parses mental activity into discrete and separable processes of sensing, thinking, and acting. In contrast, Smith and colleagues argue that the shape bias is an attentional bias that itself is the developmental product of the child's exposure to a language in which solidity and category organization by shape are highly correlated (Smith, 2000; Smith and Samuelson, 2006). This idea stems from the view of cognition that sees knowledge as embedded in process and denies a separation between sensing, thinking, and acting.

Critically, progress in this debate has been hampered by the fact that little attention has been paid to differences in the tasks and stimuli used to elicit noun generalizations in studies that purport to support one view over another. In this way, then, the word learning literature mirrors the larger developmental and cognitive science literatures both in terms of the existing disagreements concerning the nature of the cognitive system and in the need for a greater understanding of how the specifics of a task elicit knowledge in a moment. In the present paper, we examine these issues via a case study that probes similarities and differences in children's novel noun generalizations for solid and deformable things and, critically, how these differences depend on the details of the tasks that are used to elicit those generalizations. We show how an understanding of the processes that support behavior in a task yield insights into the nature of developmental changes in knowledge.

Our work is inspired by the view that knowledge is not separate from the processes that create behavior in a task. Note that this view does not deny a long-term accumulation of information based on specific experiences with objects and words. As applied to the development of the shape bias, this view suggests that as children learn individual name-object pairings, they learn a system of regularities between linguistic devices, the structure of object categories, and perceptual properties (see Smith \& Samuelson, 2006). This information accumulates over the development of the early noun vocabulary and is the "knowledge" children bring to the task of generalizing a novel name from a novel object to new instances. Thus, when we refer to "knowledge" we are referring to the child's accumulated history of experiencing naming contexts paired with objects in particular ways.

The critical question is how these prior experiences are brought to bear in the moment that a child is asked to make a novel noun generalization. In the current work, we explore this issue by first experimentally examining differences in the way children's accumulated knowledge of nominal categories is brought to bear in specific task contexts. We examine the interaction of knowledge with process by submitting the same knowledge to a model that concretely specifies critical aspects of these processes, and find that, as in the experiment with children, different behaviors emerge across tasks. Systematic manipulations of the model parameters that specify the input knowledge and processes demonstrate that the experimental pattern of re- 
sults only obtains when the inputs are processed in particular ways. This is further illustrated in simulations of developmental changes in novel noun generalization. This work thus demonstrates that changes to either the input knowledge alone or the processes alone do not capture differences in children's behavior across tasks and development-it takes a coherent pattern of change in both to yield the specific pattern of behavioral change.

\subsection{Background: naming deformable things}

A bias to attend to shape, although useful for learning nouns such as table, hammer, and key, that name solid objects in shape-based categories (Samuelson \& Smith, 1999), may not be appropriate for all nominal categories. In particular, deformable things such as paper, blankets, and towels provide an interesting test case for children's attention to shape because similarity in material may be as important to the organization of these nominal categories as similarity in shape. In fact, studies with adults suggest deformable things are organized into nominal categories based on similarity of material (Samuelson and Smith, 1999; Samuelson and Smith, 2000a). Further, material substance is often critical to what can be done with these things. For example, while both blankets and towels are likely to have a rectangular shape, it is their particular material composition that distinguishes one from the other and that influences what we do with each. Thus, children may be biased to generalize novel names for novel deformable things according to material substance.

On the other hand, many deformable things do have characteristic shapes (e.g., shirts, pants, and socks) and thus shape is an important factor in the organization of these nominal categories. Further, while deformable things can be folded, balled, squished or otherwise manipulated to alter their shape, they can be returned to their original shapes following such manipulations. In addition, analysis of the early noun vocabulary suggests that many of the categories of deformable things that children learn to name early are similar to categories of rigid things in that both are named with count nouns ("a shirt" rather than "*some shirt", Samuelson \& Smith, 1999). In the early noun vocabulary, count noun syntax is associated with categories organized by similarity in shape (Samuelson \& Smith, 1999). It is therefore also possible that the similarity in the syntactic frame associated with rigid and deformable things may direct children's attention to shape when naming deformables (Samuelson et al., 2008; Samuelson and Smith, 2000a).

Thus, there are reasons to expect that compared to rigid things, children's naming of deformables would be more variable and subject to influences of task structure. There is some indication of this in the literature. Samuelson and Smith (2000) found that 3-year-old children generalized novel names by similarity in shape in a yes/no task. In contrast, Gathercole and colleagues (Gathercole, Cramer, Somerville, \& Haar, 1995) found that 4-year-old children were more likely to attend to substance when naming deformable stimuli in a forced-choice task (see also Samuelson et al., 2008). However, direct comparison between studies is difficult due to differences in the stimuli used and the specifics of the task (i.e. whether a function or property of the exemplar was demonstrated prior to naming). Further, the use of children of different ages across studies also means that participants differed in vocabulary, which has been suggested to be a critical determinate to attention to shape in similar tasks (Gathercole and Min, 1997; Gershkoff-Stowe and Smith, 2004; Samuelson and Smith, 1999).

\subsection{Overview of this study}

The fact that some factors associated with deformables may direct children's attention to similarities in material, while other factors may direct their attention to similarities in shape provides an opportunity to probe whether and how the specifics of the task influence children's noun generalizations. Toward this end, we present an experiment that contrasts the performance of children the same age and with the same productive vocabulary across forcedchoice and yes/no noun generalization tasks with the same stimuli. Data from this experiment suggest important differences in the specifics of children's performance related to the particulars of the two tasks. We present a dynamic field model to formally specify the real-time processes that turn knowledge of nominal categories into a forced-choice or yes/no response. The model reveals constraints on the structure of the accumulated knowledge children bring to bear in these tasks. We show that the combination of the hypothesized processes and knowledge accurately captures both global qualitative differences in performance across forced-choice and yes/no tasks as well as specific quantitative differences.

Next, we quantitatively fit data from 2- to 4-year-old children from a study using the same stimuli in a yes/no task. These fits illustrate how changes in children's accumulated knowledge of nominal categories translate into developmental changes in behavior in this task. Thus, the model illustrates how an understanding of process provides insights both into what children bring to the task based on their prior history and how this accumulated prior history changes over time. The model also illustrates the dynamic nature of knowledge and it's inseparability from process, and thus, has important implications for our understanding of cognition more generally.

\section{Experiment 1}

Most of the categories of rigid things that young children learn to name early provide clear links among solidity, category organization, and syntax - solid, rigid things tend to be in categories organized by similarity in shape and tend to be named with count nouns (Samuelson \& Smith, 1999). Moreover, research suggests that children learn to exploit this regularity in novel noun generalization (Samuelson, 2002; Smith et al., 2002). In contrast, the deformable things children learn to name early do not present as regular a relationship (Gathercole et al., 1995; Samuelson and Smith, 1999; Samuelson and Smith, 2000a). Many deformable things have specific shapes but those shapes can be changed. Adults typ- 
ically judge deformable things, like nonsolid substances, to be in categories well organized by similarity in material substance, but deformable things, like rigid objects, are often named with count nouns (Samuelson \& Smith, 2000a). Given the possibility that young children could organize deformable things by either shape or material, the purpose of this experiment was to directly examine the extent to which children's noun generalizations with deformable things are influenced by the task. We collected novel noun generalizations in forced-choice and yes/no tasks from groups of children that were the same in age and productive vocabulary. We tested 2.5-year-old children because previous studies suggest developmental changes in the amount of attention to shape in noun generalization between 2 and 3 years of age (Gershkoff-Stowe and Smith, 2004; Landau et al., 1998; Samuelson et al., 2008; Smith et al., 1992), and because pilot testing suggested that children this age could effectively complete both forced-choice and yes/no versions of the task. In both tasks, we highlighted the rigidity or deformability of the exemplar object before naming it. This was done to ensure children were aware of the relevant differences in the stimuli, and because prior studies with these and similar stimuli had used this procedure (Gathercole and Min, 1997; Samuelson and Smith, 2000a; Samuelson et al., 2008).

\subsection{Method}

\subsubsection{Participants}

Thirty-two monolingual, English-speaking children $(M=31 \mathrm{~m} 13 \mathrm{~d}$, range $=28 \mathrm{~m} 16 \mathrm{~d}-32 \mathrm{~m} 24 \mathrm{~d} ; 18$ girls, 14 boys) were recruited from local county birth records. All of the children were from middle-class families. Half of the children participated in a forced-choice task ( 8 females, 8 males) and half in a yes/no task (6 females, 10 males). Children in the forced-choice and yes/no conditions did not differ in age ( $M=31 \mathrm{~m} 11 \mathrm{~d}$ and $31 \mathrm{~m} 16 \mathrm{~d}$, respectively), $t(30)=.36$, ns, or total noun vocabulary $(M=284.8$ and 293.5, respectively), $t(30)=.88$, ns, as measured by the MacArthurBates Communicative Development Inventory: Words and Sentences (MCDI; Fenson et al., 1994) - a validated parental checklist of the 680 words commonly learned by children up to 30-months of age. Data from an additional four children who participated in the yes/no condition were not analyzed due to experimental error (1), because the child did not finish (1), or due to a potential language delay (2). Informed consent was obtained from children's parent or guardian prior to the experimental session. Children received a small gift for participating.

\subsubsection{Stimuli}

Nine objects familiar to young children were used on training trials. The exemplar for these trials was a purple plastic egg. The test objects were an identical egg, a red wooden block, a plastic flower, a plastic teapot, a small basket, a rubber duck, a multi-colored miniature slinky, and a small stuffed dinosaur. All of the objects were similar in size.

Four sets of novel objects were used during the test trials (see Figure 1). Each set consisted of an exemplar and six test objects. For each set, two of the test objects were the same shape as the exemplar but were a different color and were made from a different material, two were made from the same material as the exemplar but were a different shape and color, and two were the same color as the exemplar but different in shape and material. The exemplars for sets 1 and 2 were made from rigid materials. The exemplars for sets 3 and 4 were made from nonrigid, deformable materials. The exemplar for set 1 was a $14.0 \mathrm{~cm} \times 3.8 \mathrm{~cm}$ barbellshaped piece of wood painted green with a bumpy texture. The exemplar for set 2 was an $8.3 \mathrm{~cm}$ in diameter blue clay ball with four clay pegs. The exemplar for set 3 was a $14.0 \mathrm{~cm}$ tall $\times 5.7 \mathrm{~cm}$ wide piece of yellow sponge cut into a rounded "V" shape. The exemplar for set 4 was an $11.4 \mathrm{~cm} \times 9.5 \mathrm{~cm}$ pink polygon-shaped plastic bean bag. A white tray divided into two equal sections was used to present the stimuli in the forced-choice task.

Each exemplar had two different kinds of properties that could be demonstrated. One kind of property was designed to highlight the shape of the rigid exemplars and the material of the deformable exemplars. These properties are referred to as "related" properties because they were based on the shape of the rigid exemplars and the material of the deformable exemplars. The related property for sets 1, 2, 3, and 4 were rolling, fitting into a puzzle, squishing into a cup, and folding, respectively (see Figure 1). The other kind of property was not based on the shape, color, or material of the exemplar. Thus, these are referred to as "arbitrary" properties. Because children only saw two arbitrary properties and because these properties were not based on any features of the exemplars, the same two arbitrary properties - having a small design on that glowed in the dark or a sticker on the back-were used for all four sets. Four novel names-Rel, Hux, Kiv, and Gaz - were used.

\subsubsection{Design and procedure}

The design was identical for the forced-choice and yes/ no conditions. Each child saw all four sets of stimuli. Each child saw a related property demonstrated for one of the rigid exemplars and an arbitrary property demonstrated for the other. Likewise, each child saw a related property demonstrated for one of the deformable exemplars and an arbitrary property demonstrated for the other. Which exemplar had related and which had arbitrary properties was counterbalanced across children in each condition.

Children sat across from the experimenter at a large table. Parents sat next to their child and were asked not to direct their child's responses in any way. Parents completed the MBCDI during the experimental session.

In the forced-choice condition, the experiment began with a series of training trials to familiarize the child with the task and experimental setting. On the first training trial, the child was introduced to a stuffed animal and told, "Edward is a very picky bear. He only likes things like this". The experimenter then showed the child the egg exemplar. To familiarize the child with the demonstration procedure the experimenter then said, "This is an egg, and you know what? It opens", and opened and closed the egg. The experimenter set the egg by Edward and told the child they were going to find more eggs for Edward. The experimenter gave the child 


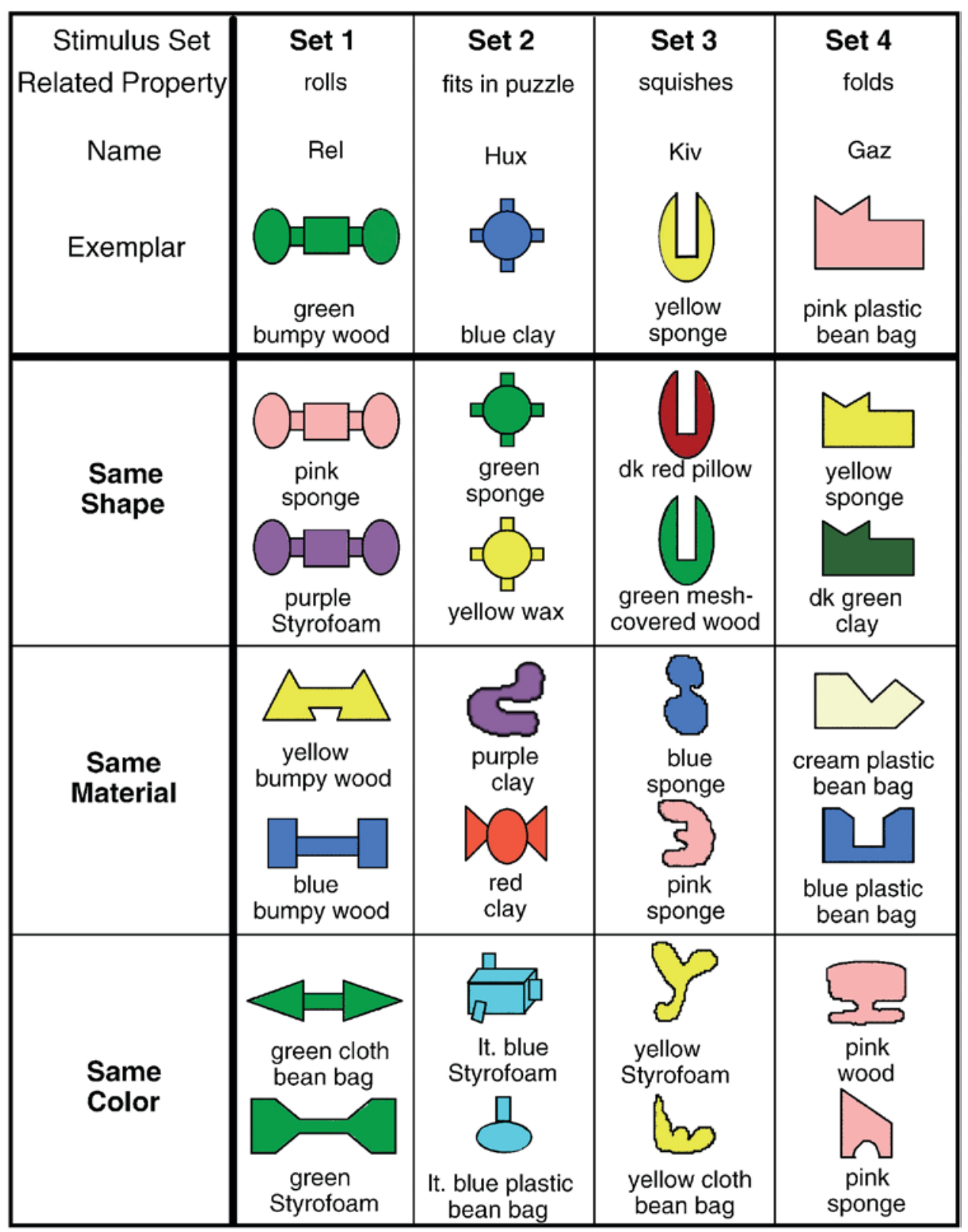

Figure 1. Stimuli used in the experiment.

two test objects to explore briefly - a second egg identical to the named exemplar, and another object that differed from the egg in shape, color, and material. The experimenter then retrieved the two test objects, placed them on the tray, said, "Remember this is an egg, and it opens (pointing to the exemplar)". She then asked, "Can you get another egg?" while sliding the tray towards the child. Children were praised if they chose the egg and corrected otherwise. The experimenter then gave the child the test objects for the next trial which proceeded in the same manner. The experimenter reminded the child that the egg opened on each training trial. The right/left position of the egg on the tray alternated across trials. To ensure that children understood the task, training trials continued until the child made four consecutive correct responses or until they had completed eight trials, at which point the experimenter continued with the test trials. All children answered at least two training trials correctly.

The test trials proceeded in the same manner as the training trials, with the exception that children's responses were neither praised nor corrected. For each of the four stimulus sets, the experimenter introduced the child to a new stuffed animal and a novel object, for example, "Nathan only likes things like this. This is a rel, and you know what? It rolls". The experimenter demonstrated the assigned property and placed the exemplar next to the animal. In order to reduce the total number of trials, and because prior studies indicated that children rarely generalized novel names to color match test objects (Samuelson et al., 2008; Samuelson \& Smith, 2000a), children did not see the color match stimuli in the forced-choice condition. Thus, on each trial the child 
was presented with a shape match and a material match. Within each set, each shape match was presented once with each material match for a total of four trials per set. Before each trial, the experimenter reminded the child of the exemplar's name and demonstrated property (e.g., "Remember, this is a rel and it rolls"). After the child completed the four trials for one set, they were allowed to choose a sticker to take home before the experimenter moved on to the next set. Children's choices were coded offline from videotape by coders unaware of the experimental hypotheses. Intercoder reliabilities were obtained for $25 \%$ of the sessions using exact percent agreement. Agreement between the two coders was $94 \%$. All disagreements were resolved by review of the videotapes.

As in the force choice condition, the yes/no condition began with training trials. The same training stimuli were used. On the first training trial, the child was introduced to a stuffed animal and told that the animal only liked eggs and was shown a plastic egg. The experimenter demonstrated that the egg opened and then brought out a series of test objects one at a time and, for each, asked, "Is this an egg?" As in the forced-choice condition, the experimenter reminded the child that the egg opened before each test trial. During training, the experimenter praised the child for correct responses and corrected incorrect responses. Each child was presented with up to eight randomly ordered training trials, which included at least two presentations of the identical egg. To ensure that children understood the task, we required that children correctly answer four consecutive training trials in order to be included in the analysis (c.f., Samuelson \& Smith, 2000a). All children met this criterion.

The test trials proceeded in the same manner as the training trials with the exception that the experimenter did not praise or correct the child during these trials and different stimulus sets were used. The experimenter introduced the child to a stuffed animal, told the child the animal only "wants things like this", named the exemplar and demonstrated the property for the child before placing the exemplar by the animal. Then the experimenter presented each test object one at a time. Before presenting each test object, the experimenter reminded the child of the exemplar's property. For example, the experimenter would say "Remember, this is a rel and it rolls", and then present a test object and ask, "Is this a rel?" After all six test objects for a set were presented, the experimenter moved on to the next set. As in previous studies using this task, we required that children say "no" to at least one test object during the experimental trials in order to be included in the final analyses (c.f., Samuelson \& Smith, 2000a). All children met this criterion. This provided an additional check that children understood the task. The order of exemplars and test objects was randomly determined and counterbalanced across children. Children's yes/no responses were recorded by the experimenter during the experiment.

\subsection{Results}

The left panels of Figure 2 show the proportion of shape choices in the forced-choice task for deformable (top panel) and rigid (bottom panel) exemplars. The right panels of the figure show the proportion of yes responses to each of the three test objects in the yes/no task for the deformable (top panel) and rigid (bottom panel) exemplars. Analyses revealed no effects of relatedness (i.e., whether an arbitrary or related property was demonstrated); thus, the figure collapses across this factor. The data in the figure indicate that children's attention to shape when naming deformable exemplars was influenced by the task, while attention to shape when naming rigid exemplars remained uniformly high regardless of the task. We first examined the patterns of noun generalization within each task using ANOVAs. We then performed a set of analyses comparing data from the two tasks directly.

\subsubsection{Forced-choice task}

As can be seen in the left panels of Figure 2, children were more likely to generalize names for deformable things by similar material than by similar shape, but systematically generalized novel names for rigid things by similar shape. This was confirmed by a rigidity (rigid vs. deformable) $\times$ relatedness (related vs. arbitrary) repeated measures ANOVA in which both factors were within subjects. This analysis revealed a significant main effect of rigidity $F(1,15)=29.25$, $p<.0001, \eta_{\mathrm{p}}{ }^{2}=.66$, but no other main effects or interactions. $t$-Tests against chance confirmed that children chose shape-matching test objects at levels significantly below chance when exemplars were deformable, $t(15)=-2.145$, $p<.05, d=.24$, but above chance when exemplars were rigid, $t(15)=5.13, p<.001, d=1.037$.

\subsubsection{Yes/no task}

As can be seen in the right panels of Figure 2, children were more likely to generalize novel names for both deformable and rigid exemplars by shape in the yes/no task. Children's yes responses were entered into a rigidity (rigid vs. deformable) $\times$ relatedness (related vs. arbitrary) $\times$ test object (shape, color, material) repeated measures ANOVA. There were significant main effects of rigidity, $F(1,15)=6.32, p<.05, \eta_{\mathrm{p}}{ }^{2}=.30$, and test object, $F(1,30)=28.36, p<.0001, \eta_{\mathrm{p}}{ }^{2}=.65$, and a significant rigidity $\times$ test object interaction, $F(1,30)=7.36, p<.01, \eta_{\mathrm{p}}{ }^{2}=.33$. There were no significant main effects or interactions involving relatedness. Thus, the data were collapsed across relatedness and further analyzed via simple effects tests split by rigidity.

For the deformable exemplars, there was a significant effect of test object, $F(1,30)=17.12, p<.0001, \eta_{\mathrm{p}}^{2}=.59$. Follow-up Fisher's PLSD tests revealed that the proportion of yes responses to shape-matching test objects was significantly higher than the proportion of yes responses to material-matching test objects which in turn, was higher than the proportion for color-matching test objects. For the rigid exemplars, there was also a significant effect of test object, $F(1,30)=27.53, p<.0001, \eta_{\mathrm{p}}{ }^{2}=.65$. Follow-up Fisher's PLSD tests revealed that the proportion of yes responses to the shape-matching test objects was higher than the proportions for the material- and color-matching test objects, which did not differ significantly. 
Fecioh Cdecro
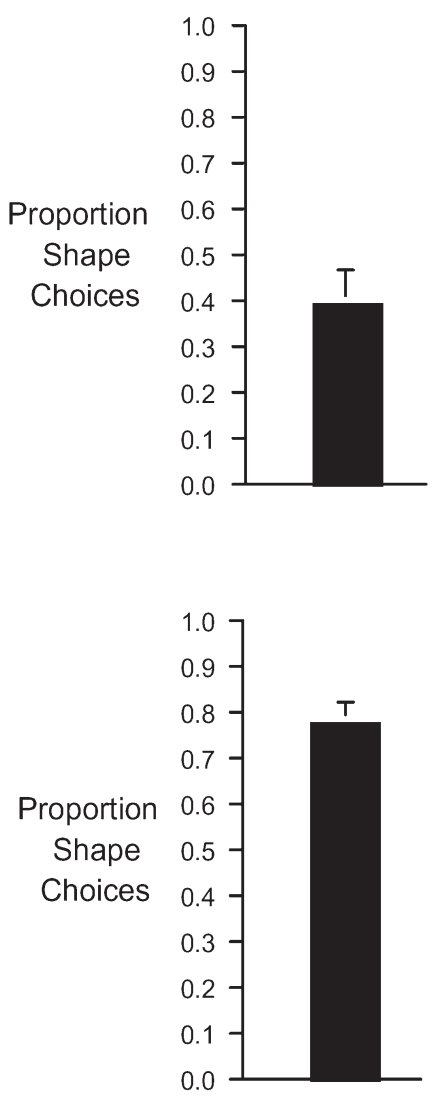

Deformable

Yes/ No

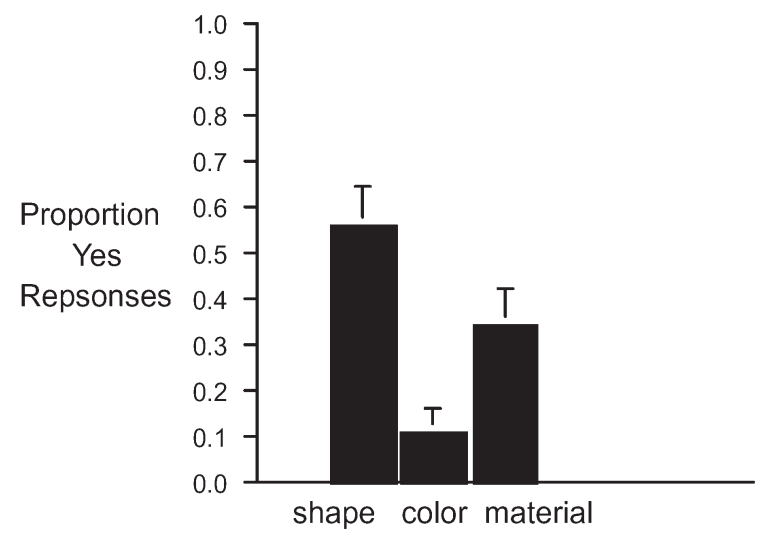

Rigid

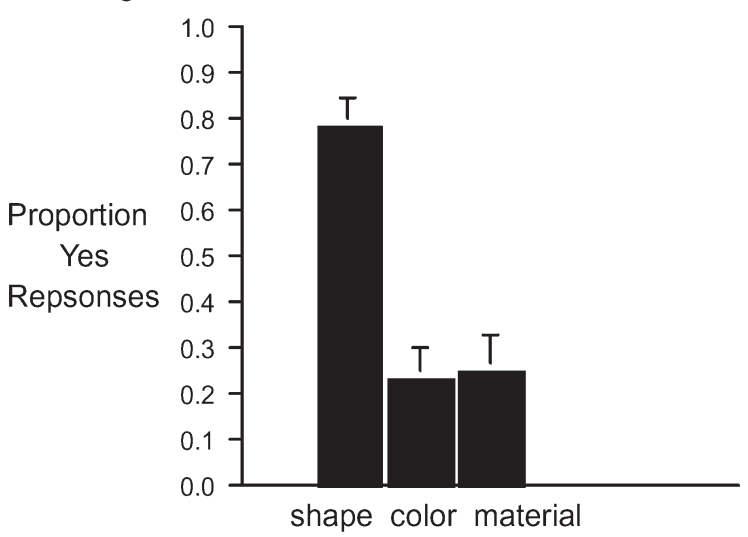

Figure 2. Data from the experiment. The left panels show the proportion of shape choices for deformable and rigid exemplars following demonstrations of related and arbitrary properties in the forced-choice task. The right panels show the corresponding proportions of yes responses to the shape-, color-, and material-matching test objects in the yes/no task. There were no effects of relatedness so the figure collapses across this factor.

\subsubsection{Comparison across tasks}

Analyses of responses in each task suggest that 2.5-yearold children generalized novel names for rigid things by shape in both the forced-choice and yes/no tasks, but only systematically generalized novel names for deformable things by material in the forced-choice task. To examine the difference in responding across tasks more directly, we compared the yes/no and forced-choice data after converting the yes/no data to proportion of shape choices via the Luce choice rule (Luce, 1963). Specifically, for individual children the proportion of shape choices for a given stimulus set was taken to be equal to the proportion of yes responses to shape-matching test objects divided by the sum of the proportion of yes responses to shape- and material-matching test objects. ${ }^{1}$ We collapsed over relatedness in this analysis because the ANOVAs showed no significant effects of this factor. Thus, the proportion of shape choices for the deformable and rigid sets was taken to be equal to the average proportion of yes responses to shape-matching test objects

1. One child in the yes/no task said "no" to all the deformable test objects. This would result in a proportion shape score of 0 by the Luce choice rule, even though the child did not say "yes" to any of the material-matching test objects. Thus, to be conservative, we opted to not include data from this child in this analysis. divided by the sum of the average proportions of yes responses to shape- and material-matching test objects. These data are shown in Figure 3, along with the corresponding data from the forced-choice task.



Figure 3. Comparison of performance in the forced-choice and yes/ no tasks of the experiment. Yes/no data were converted to proportion shape choices via the Luce choice rule. 
Table 1. Numbers of individual children in the forced-choice and yes/no conditions of Experiment 1 whose proportion of shape choices was below chance $(<.50)$, at chance levels $(<.50)$ or above chance $(.<.50)$ for the deformable and rigid stimulus sets. Results of chi-square tests of homogeneity of proportions comparing the patterns of responding across the two tasks for each stimulus set are also provided.

\begin{tabular}{llll}
\hline & \multicolumn{3}{c}{ Condition } \\
\cline { 2 - 4 } & & Forced-choice & Yes/no \\
\hline Deformable & $<.50$ & 6 & 2 \\
Proportion shape & $=.50$ & 9 & 4 \\
& $>.50$ & 1 & 9 \\
& & $\chi^{2}(2)=11.23, p<.01$ & \\
Rigid & & & 0 \\
Proportion shape & $<.50$ & 2 & 4 \\
& $=.50$ & 1 & 12 \\
& $>.50$ & 13 & \\
& & $\chi^{2}(2)=3.80, \mathrm{~ns}$ & \\
\hline
\end{tabular}

The total number of children reported for the yes/no task with deformable stimuli is not equal to 16 because one child said no to all the deformable test objects.

As is clear in the figure, the proportion of shape choices made when a rigid exemplar was named did not differ across task. Importantly, however, the proportion of shape choices made when a deformable exemplar was named differed markedly across tasks. We analyzed this data at the level of individual children. Table 1 provides the numbers of individual children in each condition whose proportion of shape choices was below chance $(<.50)$, at chance $(=.50)$ or above chance $(>.50)$ for both the deformable and rigid stimulus sets. As is clear in the table, with deformable stimuli there were more children whose proportion of shape choices was at or below chance in the forced-choice condition than the yes/no condition. In contrast, with the rigid stimuli the numbers of children whose proportion shape choices was above chance were almost identical across tasks. Chisquare tests of homogeneity of proportions revealed a significant difference across conditions with deformable stimuli, $\chi^{2}(2)=11.23, p<.01, w=.60$, but no significant difference with rigid stimuli, $\chi^{2}(2)=3.80$, ns.

\subsection{Discussion}

These results indicate that children equated for age and productive vocabulary generalize names for deformable stimuli differently when presented with a forced-choice compared to a yes/no task. Specifically, data from the forcedchoice task suggest that 2.5-year-old children think that rigid things are named by similarity in shape whereas deformable things are named by similarity in material substance. In contrast, data from the yes/no task suggest children this age do not distinguish between rigid and deformable things in naming, and generalize names for both kinds by shape similarity. In this way, then, data from the two tasks lead to different conclusions about what children know about how deformable things are named. Given that the same stimuli were used in the two tasks and that groups were equated for age and vocabulary level, these data suggest that differences in the processes that bring children's accumulated vocabulary knowledge to bear in each task are critical to determining the specifics of their performance.

There are two critical differences between the forcedchoice task and the yes/no task that seem likely to influence children's behavior. First, in the forced-choice task, children see the two test objects at the same time in close proximity (i.e., on the same tray), whereas in the yes/no version of the task, children see each test object one at a time. Thus, children can make direct comparisons between the test objects in the forced-choice task, but must make these comparisons in memory (if at all) in the yes/no version of the task. Second, the nature of the forced-choice task is to force the child to pick one object to the exclusion of the other, whereas in the yes/no version of the task, the child is free to say yes (or no) to all of the test items individually.

These differences have important implications for how children's prior history of perceiving and acting is brought to bear in a moment in time, that is, for how their accumulated knowledge of nominal categories is brought to bear in different novel noun generalization tasks. For example, a relatively small bias to favor similarity in material may be magni.ed when children are forced to pick between a test object that matches an exemplar in shape and one that matches it in material. Likewise, a relatively weak appreciation of the importance of shape for deformable things may be more robust in a yes/no task because judgments of the similarity of shape and material test objects are made independently. To probe these issues in greater detail, we implemented these task differences in a dynamic field model and simulated performance in the forced-choice and yes/no versions of the novel noun generalization task.

\section{Quantitative modeling of performance across tasks}

The general goal of these simulations was to examine the possibility that different patterns of behavior can arise in the forced-choice and yes/no tasks even when the same stimuli are used and the same knowledge is accessed. If this is the case, it suggests that some of the differences observed in the previous experiment may arise from the specifics of the real-time processes that combine perceptual inputs with long-term memory of how stimuli are categorized to create responses in novel noun generalization tasks. The specific goal of our modeling efforts was to quantitatively fit the data from the experiment, that is, to produce the particular amounts of attention to shape and material we found in 2.5-year-olds in a model that captures the processing differences underlying the forced-choice and yes/no tasks. Note that we did not simulate the demonstrations of related and arbitrary properties because this aspect of the task had little influence on children's performance. Likewise, we did not simulate performance with the color-matching stimuli because these stimuli were not included in the forced-choice task.

In what follows, we first present a general overview of our dynamic field model, including qualitative results from single representative simulations of performance in each 
task. We then discuss the selection of the particular parameters we used. Finally, we present quantitative results from our simulations of the data from the experiment, followed by a discussion of the implications of this modeling work. The details of the model, including the model equations, can be found in the Appendix.

\subsection{The model}

The starting point for the model is work by Spencer and colleagues modeling the dynamics of responses in forcedchoice and discrimination (same/different) tasks (Johnson, Spencer, \& Schöner, 2008; Simmering, Spencer, \& Schöner, 2006; Spencer, Simmering, \& Schutte, 2006). Our model, pictured in Figure 4A, is an extension of the Dynamic Field Theory (DFT), a general theory of spatial cognition and visual working memory (see Johnson et al., 2008; Spencer, Simmering, Schutte, \& Schöner, 2007). The current work builds on prior theoretical, computational, and empirical work using DFT to capture real-time and developmental change in tasks ranging from spatial memory to change detection to habituation in infancy. Note that by "real-time" we are referring to the time scale of processes that generate individual responses within a trial. Previous work has mapped the real-time generation of peaks in similar dynamic neural eld models to the generation of neural representations in visual cortex and motor cortex (Bastian, Riehle, Erlhagen, \& Schöner, 1998; Bastian, Schöner, \& Riehle, 2003; Jancke et al., 1999). However, this is the .rst application of DFT concepts to task-specific behavior in a wordlearning context (for related work, see Faubel \& Schöner, 2008).

The model consists of an input field (top field in panel A) and decision field (bottom field Panel A). The $x$-axis of each field shows a set of neurons arranged by "similarity." Neurons that are tuned to respond to stimuli that are similar overall (i.e., with receptive fields tuned to similar values along particular feature dimensions) would be close neighbors along this dimension, while neurons that are tuned to respond to stimuli that are dissimilar overall would be far from one another along this dimension. The activation of each neuron is plotted along the $y$-axis. Time is shown along the $z$-axis as the sequence of events in a single trial unfolds. Note that many models of similarity and object categorization compute similarity as the distance between the high-dimensional representations generated by, say, the presentation of two objects (e.g., Kruschke, 1992; Nosofsky, 1987; Samuelson, 2002). Our use of similarity capitalizes on this idea, that is, we assume a system that can represent similarity in this way and pass metric input regarding the distance between two items to the decision field probed here (for an additional discussion of the concept of "similarity" in dynamic field models, see the Section 5 and Faubel \& Schöner, 2008). Importantly, we assume that the metric similarity input to the model reflects similarity in the context of a naming task (see discussion of parameters below). Note also that the strength of inputs captures the salience of each item. Salience can be affected by multiple factors such as long-term learning about which features of objects are important for word learning, or factors such as whether an item is within graspable space.
The stimuli were presented to the model via the input field. Inputs took the form of Gaussians representing the exemplar (leftmost peak) and two test objects, positioned according to their relative similarities (see Section 3.2). The number of test objects presented to the decision field differed according to the task (see below). The exemplar was present throughout the trial in both tasks, but its activation was lower because it is farther from the child.

The time-dependent processes (i.e., the dynamics) that determine the model's response occur in the decision field. Neurons in this field interact according to a local excitation/ lateral inhibition function, a form of interaction common in neural models of cortical function (e.g., Durstewitz, Seamans, \& Sejnowski, 2000). This means that neurons close to one another along the similarity dimension excite one another while neurons far apart inhibit each other. The local excitation/lateral inhibition function allows the network to form stable peaks of activation that represent behavioral decisions to, for instance, select a particular input in a forcedchoice task. It is also possible, however, that the model will fail to form an activation peak. Whether this occurs depends on the similarity of inputs, the strength of neural interactions (i.e., the details of the interaction function), and the resting level of the neurons in the field. The critical differences between the forced-choice and yes/no versions of the novel noun generalization task emerge from differences in the strength and time structure of the inputs and from the interactive dynamics of the decision field. These differences are described below.

\subsubsection{Dynamics of the forced-choice task}

In the forced-choice version of the novel noun generalization task, the child is presented with two test objects at the same time and encouraged to pick one on each trial. Thus, children have the opportunity to compare the test objects directly and have to make a relatively constrained responseselect one object over the other. As can be seen in the top field of Figure 4A, on each trial the model is also presented with two test objects at the same time and encouraged to pick one-that is, to form a peak of activation centered at one input or the other. To capture the highly selective nature of this task, we made the inputs strong and the decision field competitive (i.e., neurons interact strongly). This allows for a detailed "comparison" between stimuli. Figure 5 shows the interaction kernels for each task (see Table 2 for specific parameter values). As can bee seen in the figure, the parameters that structured the interaction dynamics in the forced-choice task were such that the decision field started relatively far from threshold (see $h_{i}$ in Table 2) and had strong levels of inhibition (see $w_{i F C}$ in Figure 5) with strong excitatory interaction ( $w_{e F C}$ in Figure 5$)$ and strong inputs $(\kappa)$. Consequently, the decision field reflected the inputs strongly and was highly competitive. When the tray was moved forward and the resting level boosted (see $h_{b}$ in Table 2), competition increased and the field was forced to make a selection. Importantly, this set of parameters put the field in a regime in which the peak that formed was centered at the location of one of the two test objects, rather than at an indeterminate location or at the location of the exemplar. 


\section{A Forced Choice Response}



B "Yes"Response
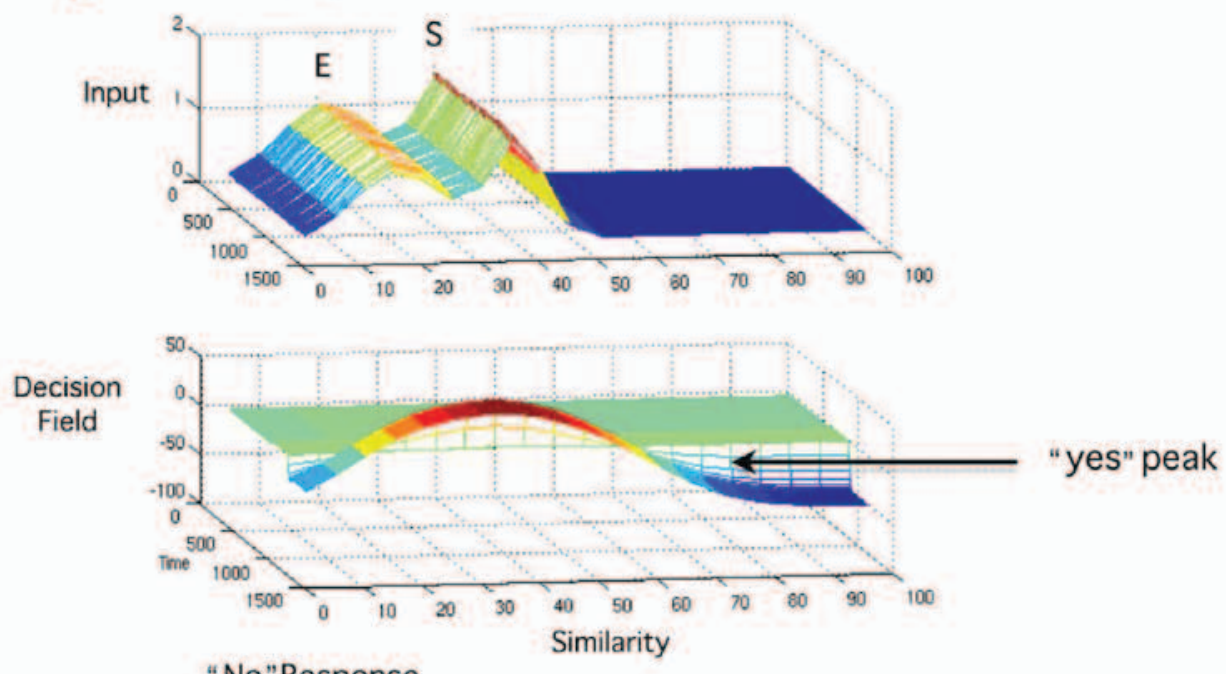

"No"Response



Figure 4. Dynamic field model used to simulate differences in performance in forced-choice and yes/no tasks. Model consists of an input field (top field in Panel A) and decision field (bottom field Panel A). The $x$-axis of each field represents a set of neurons arranged by "similarity". Activation of each neuron is plotted along the $y$-axis. Time is shown along the $z$-axis as the sequence of events in a single trial unfolds. Panel A depicts a forced-choice trial. The top two fields in Panel B depict a yes/no trial with similar inputs, the bottom two fields, a yes/no trial with dissimilar inputs. See text for further details. 


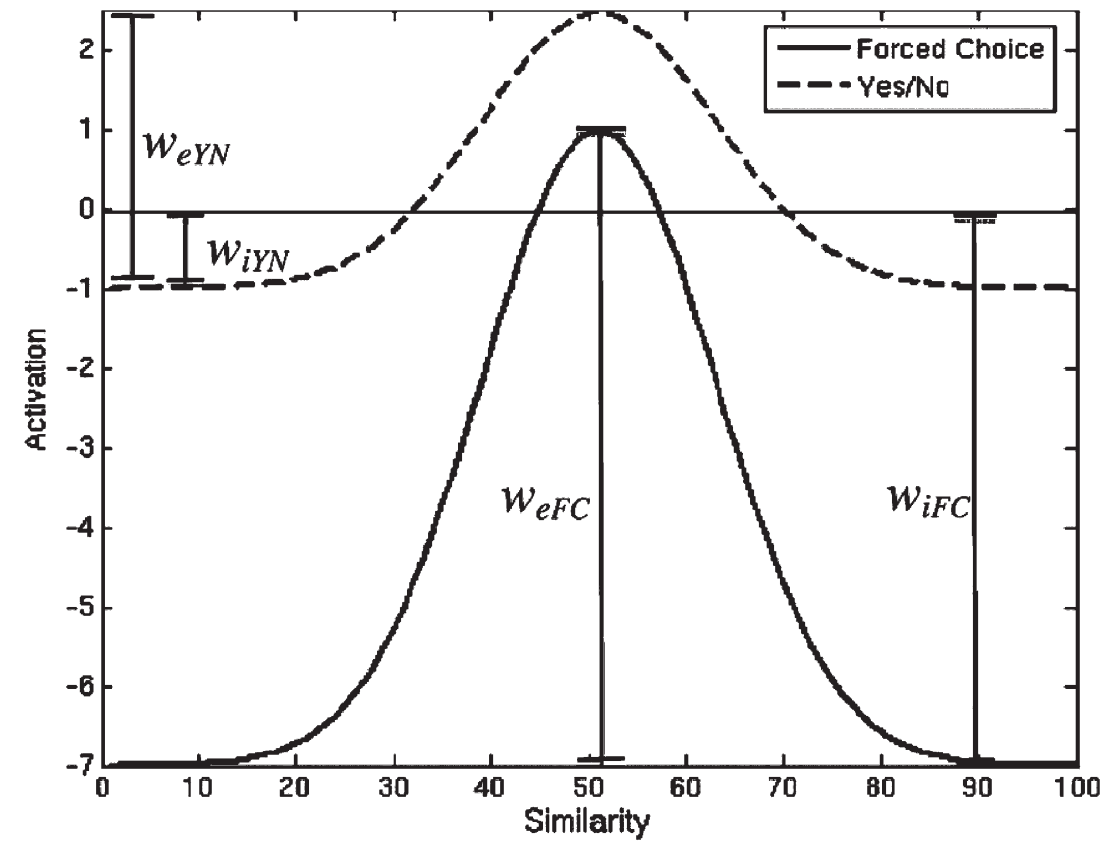

Figure 5. Interaction functions for the decision field when the model performed the forced-choice (solid line) and yes/no (dashed line) tasks. The solid line at 0 marks the difference between excitatory interactions and inhibitory interactions. Bars indicate magnitude of parameter values given in Table 2. See text for details.

Table 2. Parameters used in model simulations. Parameters in the upper half of the table structured the interactive dynamics in the response field for the forced-choice and yes/no tasks. Parameters in the lower half of the table specified the Gaussian inputs for the deformable and rigid test sets. The three values of each parameter in this section correspond to the exemplar, shape-matching test object, and material-matching test object, respectively. See text for discussion.

\begin{tabular}{lcccc}
\hline & & Parameters for interactive dynamics \\
\hline & & Forced-choice & Yes/no & Description \\
\hline Interaction inhibition & $\left(w_{i}\right)$ & 7.0 & 1.0 & How much neurons inhibit each other \\
Interaction strength & $\left(w_{e}\right)$ & 20 & 8.68 & Amount of interaction between neurons \\
Initial H level & $\left(h_{i}\right)$ & -15.0 & -5.0 & Resting level of field \\
Response H level & $\left(h_{b}\right)$ & -2.0 & -2.365 & Field resting when response requested \\
Input gain & $(\kappa)$ & 6.7 & 1.0 & Strength of input to decision field \\
& & \multicolumn{5}{c}{ Input parameters } \\
\end{tabular}

Deformable (exemplar, shape, material)

$\begin{array}{lll}\text { Input width } & \left(\sigma_{\mathrm{D}}\right) & 60,20,22 \\ \text { Input centers } & \left(x_{\mathrm{CD}}\right) & 21,41,56 \\ \text { Input strength } & \left(A_{\mathrm{D}}\right) & 1.0,1.3163,1.3623\end{array}$

Rigid (exemplar, shape, material) Input width

Input centers

$\left(\sigma_{\mathrm{R}}\right)$

Input strength

$\left(x_{\mathrm{CR}}\right)$

$(60,20,26)(.80)$

$21,41,56$

$1.0,1.3690,1.3670$

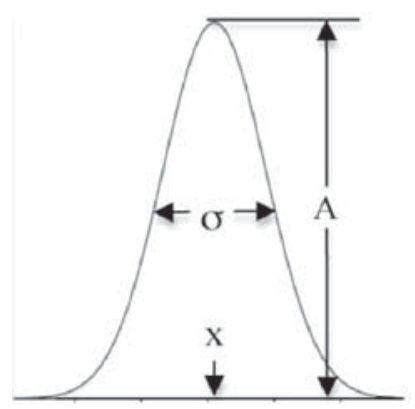

The course of events as a single forced-choice trial unfolds can be seen in the bottom field of Figure 4A. At the start of the trial (back of figure), all three inputs (pictured in the input field at the top) are fed into the decision field (lower field in panel A). The decision field is given a boost of activation at the point in the trial corresponding to when the tray with the test objects is 
slid towards the child and a request for a response is made. In the model, this change increases both competition among the neurons and the overall activation in the field, thereby forcing the creation of a single, stable peak centered at one of the test objects. As can be seen in the figure, on this trial the model formed a peak at the location of the material-matching test object; thus it "picked" this test object.

\subsubsection{Dynamics of the yes/no task}

In the yes/no version of the novel noun generalization task, the child is presented with each test object individually and must make a yes or no response for each. Consequently, the judgments children make concerning each test object are relatively independent in this task - children can say "yes" or "no" to each test object. Overall then, the decisions children make in the yes/no task are less constrained and are more sensitive to the similarity of an individual test object and the exemplar. As can be seen in the top field of Figure $4 \mathrm{~B}$, the model was also presented with one test object at a time and made an independent judgment of each. The formation of a stable peak in the decision field represented a "yes" response and the failure to form a stable peak, a "no" response. To capture the greater reliance on overall similarity in the yes/no task, we made neurons in the decision field more excitable and less competitive (see $w_{e Y N}$ in Figure 5), thereby allowing more blending across stimuli with good sensitivity to overlap in the input. In the yes/no task, the decision field started closer to threshold $\left(h_{i}\right)$ with weaker inhibition (see $w_{i \Upsilon N}$ in Figure 5) and weaker inputs ( $k$ ). Consequently, inputs (in this case, only one test object and the exemplar) could blend, and the slight boost in the resting level $\left(h_{b}\right)$ at the time a response was requested allowed for the formation of a stable peak of activation provided there was enough overlapping activation.

The top two fields of Figure $4 \mathrm{~B}$ show a simulation of a trial where there is relatively high similarity between the exemplar and the test object, while the bottom fields show a simulation with relatively low similarity. As can be seen in Figure $4 \mathrm{~B}$, when the inputs are close together, the locally excitatory interactions among neurons in the decision field cause the activation associated with these stimuli to blend and increase over time, ultimately combining to form a single activation profile. If this activation reaches a critical threshold - an activation level above 0 -the model forms a self-sustaining peak and responds "yes" (second field from top in Figure 4B). As can also be seen in the figure, however, when the inputs are farther apart, activation patterns do not blend in the decision field. Thus, no above-threshold peak is formed and the model responds "no" (bottom field in Figure $4 B)$.

\subsubsection{Summary of qualitative results}

Considered together, the simulations in Figure 4 highlight that the details of the processes that bring knowledge to bear in a task have a critical influence on the specifics of the resulting performance. In particular, note that the exact same inputs were used in the simulations presented in panels $A$ and $B$ of Figure 4. In the forced-choice task, the model picked the material-matching test object which was slightly stronger. How- ever, when this same test object was presented in the yes/ no task, the fact that this test object was relatively far from the exemplar meant that the model failed to generate a selfsustaining peak and responded "no". In contrast, when the input representing the shape-matching test object was presented in the yes/no task, the shared similarity caused a selfsustaining peak to form and the model responded "yes". Thus, given the exact same inputs in the two tasks, the model generalized the names differently, demonstrating a material bias in the forced-choice task, but a shape bias in the yes/no task. The central question, then, is whether these qualitative insights can be extended to provide a quantitative account of the findings from the experiment.

\subsection{Method}

\subsubsection{Model parameters}

Table 2 lists the model parameters organized as "interaction" parameters that structured the interactive dynamics in the decision field for the forced-choice and yes/no tasks, and "input" parameters that specified the Gaussian inputs for the deformable and rigid stimulus sets. We discuss constraints on parameter selection below.

Parameters for interactive dynamics: Selection of the values for the interaction parameters was constrained by the theoretical proposals reviewed above regarding the nature of the decision dynamics in the forced-choice and yes/no tasks. That is, the parameters that specified the interactive dynamics for each task were selected to implement the hypothesis that the forced-choice task requires children to be highly selective while performance in the yes/no task depends more on the overall similarity between an individual test object and the exemplar. If our selected parameters then capture differences in children's performance between tasks, this will support our theoretical proposal about the critical processes that underlie performance in the two tasks.

Note that in the forced-choice task, the model was required to choose one of the two test objects by building a peak of activation in the decision field centered on the location of one of the two input peaks. In contrast, the particular response made in the yes/no task depends on whether or not a stable peak of activation forms, not on the exact location of that peak. Thus, we did not require that the parameters for the yes/no task place the field in a regime that constrained peak formation to the location of an input. We did verify, however, that peaks did not form outside the range of the inputs to ensure that they were the result of the interaction of the inputs, not spurious peaks created by noise.

As can be seen in Table 2, five parameters that determined the competitive nature of the interactions were varied across tasks. Conceptually, these parameters specify how children's attention is modulated by the specifics of the task (see Section 2.3 for further details).

Input parameters: Selection of the input parameter values was constrained by the goal of presenting the same rigid and deformable inputs in the two tasks and by the necessity of finding reasonable parameters given what is known about children's attention to shape and material in novel noun generalization tasks. In addition, we wanted to 
be able to manipulate the inputs to examine possible constrains on the interface between children's knowledge and task performance (see Section 2.3). The inputs that represented the exemplar, shape-matching, and material-matching test objects for the deformable and rigid sets were represented by two sets of three Gaussians. Each Gaussian was specified by three parameters: (1) input center $(x),(2)$ input width $(\sigma)$, and (3) input strength $(A)$. The final parameter values are given in Table 2 . In the table, the values given for each parameter are for the exemplar, shape-matching test object, and material-matching test object, respectively. Differences in relative position, strength, and width of the inputs within and across input sets can be taken to capture overall similarity, strength, and precision of the representational states, respectively, that were created when children viewed the stimuli in the context of a novel noun generalization task.

As can be seen in the table, the input width values for test objects for both the deformable $\left(\sigma_{\mathrm{D}}\right)$ and rigid $\left(\sigma_{\mathrm{R}}\right)$ sets were not as broad as the exemplar in either set. This is because the test objects were always presented closer to the children than the exemplar and were the focus of children's attention. The fact that the shape-matching test object was a bit narrower than the material-matching test object in each set fits with data suggesting children make distinctions in noun generalization based on shape similarity before they make similar distinctions based on material similarity (Gathercole and Whitfield, 2001; Landau et al., 1988; Samuelson and Smith, 1999). Note that for the rigid stimuli, the input width values were scaled by .80 . This fits with the fact that rigid things generally have more precise featural characteristics than deformable things.

The same input center values were used for the both the deformable $\left(x_{\mathrm{CD}}\right)$ and rigid $\left(x_{\mathrm{CR}}\right)$ sets. In both sets, the shapematching test object was more similar to the exemplar (i.e., their centers were closer) than the material-matching test object. For the deformable set, the greater similarity of the shape-matching test object to the exemplar fits with the fact that deformable things have characteristic shapes that they return to following perturbations. Similarly, for the rigid set, the greater similarity of the shape-matching test object fits the fact that rigid things maintain their shape over transformations. The greater similarity of shape-matching test objects to the exemplar in both sets also fits with data suggesting that children have learned an association between the importance of shape similarity and naming tasks such as the ones we are modeling (Landau et al., 1988; Samuelson, 2002; Smith and Samuelson, 2006; Smith et al., 2002).

The input strengths were slightly different for the two kinds of stimuli. Specifically, for the deformable sets, the material-matching test object had a slightly higher strength than the shape-matching one. This reflects the importance of material for what can be done with deformable things, especially in a task in which a demonstration is used to highlight the material composition of some of the stimuli. The two test objects were more similar in strength for the rigid set, though the shape-matching test object was slightly stronger to reflect the importance of shape for what can be done with rigid things. It is also important to emphasize that the par- ticular strength values in Table 2 were multiplied by the input gain $(\kappa)$ for each task. Thus, the small differences in the strength of the material- and shape-matching test objects within a set were amplified in the forced-choice task relative to the yes/no task due to the greater input gain.

Figure 6 depicts the inputs for the deformable and rigid sets that result from the final parameters. This figure highlights the similarity of the inputs across sets. Nevertheless, we will see that these small differences had a substantial impact on the model's performance in the context of the interactive dynamics of the decision field.

\subsubsection{Simulation details}

Simulations were conducted in MATLAB (Mathworks Inc.) on a PowerMac G5 with dual $2.7 \mathrm{GHz}$ processors (the MATLAB code is available from the first author on request). The dynamic field equation was integrated using the Euler method. Each run of the model in each task simulated a single trial. A network with 101 neurons was used for all simulations. Trials began with the field at the level specified by $h_{i}$. The inputs were then presented for 610 time steps. Following this, the resting level was boosted to the level specified by $h_{b}$. The response was determined after an additional 500 time steps. The response in the forced-choice task was found by taking the location of the maximum activation in the decision field at the end of the simulation and determining which of the two inputs it was closest to. Individual simulations verified that the peaks that formed were at the exact location of one of the test objects. For the yes/no task, the response was taken as a "yes" if the field formed a stable peak and a "no" otherwise (responses were checked to ensure that peaks always formed within the range of the inputs). We conducted 200 repetitions of individual trials from each task with each stimulus set (deformable and rigid), and verified that the model converged on similar values across 10 sets of 800 simulations ( 200 repetitions $\times 2$ tasks $\times 2$ stimulus sets).

\subsection{Results and discussion}

We calculated the mean number of times the model selected the material- or shape-matching test object in the forced-choice task as well as the mean number of "yes" and "no" responses in the yes/no task for the deformable and rigid stimulus sets. The data from the model simulations along with the corresponding data from the experiment are presented in Figure 7. As is clear in the figure, the model fit the experimental data extremely well. To quantify the fit, we calculated the root mean squared error (RMS) across the two stimulus sets and two tasks. This value was .02 for the pictured simulation. We also examined the average RMS for the 10 sets of 800 simulations we conducted. The average was .04 with a standard deviation of .014 and a range of .02-.06. Thus, the model's performance shown in Figure 7 was quite robust.

Like the 2.5-year-old children in the experiment, the model generalized novel names for deformable things by material in the forced-choice task but by shape in the yes/no task, and generalized novel names for rigid things by shape in both tasks. The excellent fit of the model to the data even though we used the same stimulus sets across tasks thus 


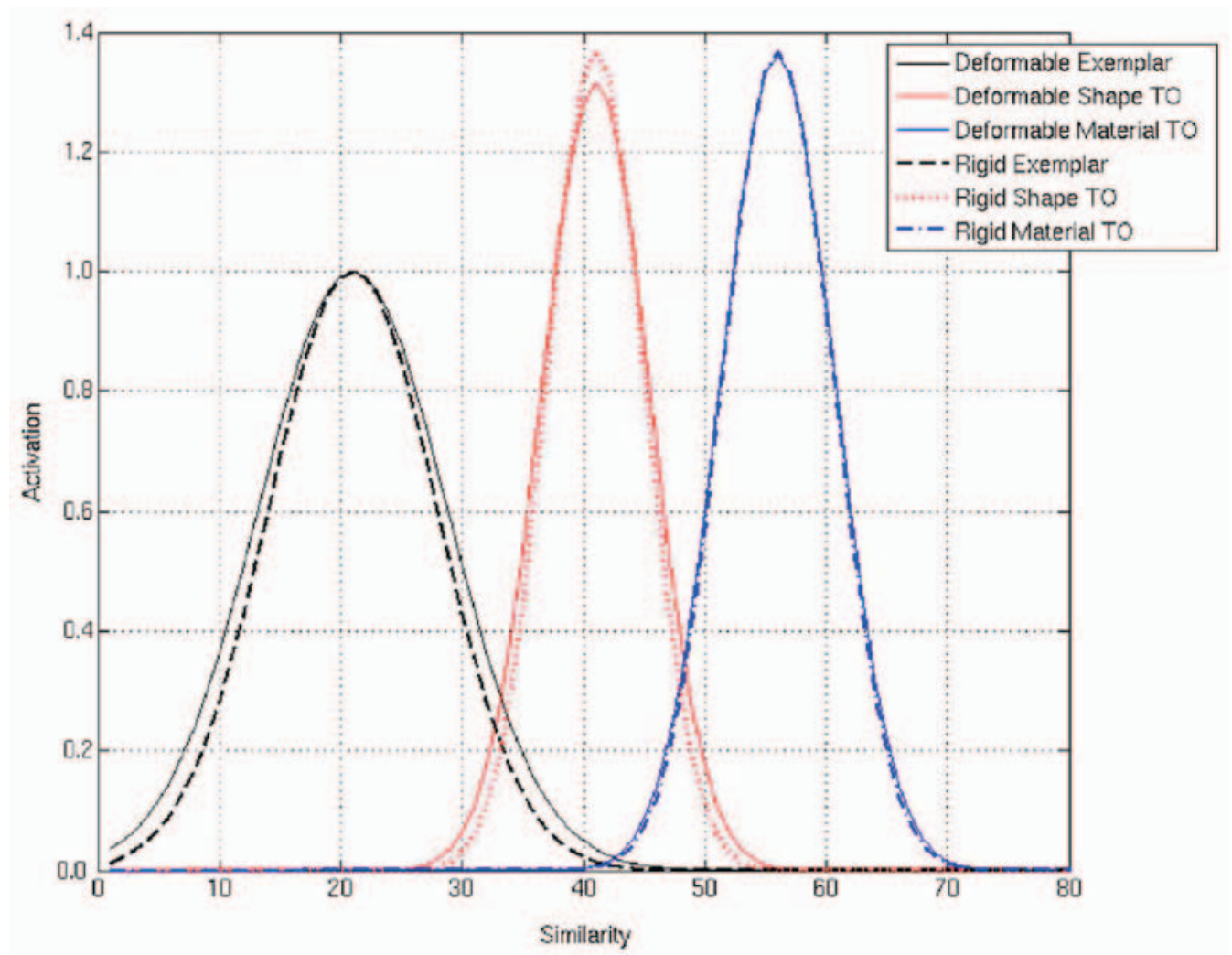

Figure 6. Model inputs for the deformable and rigid sets that result from the final input parameters. Relative overall similarity is represented along the $x$-axis, activation along the $y$-axis. Solid lines represent deformable inputs, dashed lines represent rigid inputs. Each set of inputs includes an exemplar (leftmost peaks), a shape-matching test object (center peaks), and a material-matching test object (rightmost peaks).

confirms the importance of the task dynamics in shaping children's behavior when generalizing novel nouns. These simulation results support the idea that the way children are asked to generalize novel names can create different patterns of performance even when the same accumulated vocabulary knowledge is accessed.

The robust fit of the dynamic field model to the data may lead some to question whether the model is actually too general and whether it could "model anything". The answer is no. The dynamic field equations capture strong theoretical assumptions, for example, that input is localized, that the interaction functions are homogeneous and symmetrical, and that inputs can be superimposed. Thus, simulation of any arbitrary input-output mapping is precluded by the internal structure of the DFT. Put differently, the DFT implements a form of nonlinear decision making where only the right convergence of inputs and interaction dynamics will put the field into a state that creates a stable peak. Thus, there are real constraints on processing in this model ensuring that it cannot capture just any input-output mapping.

Another critical question, however, is whether the details of the quantitative simulations are meaningful in the context of the extant literature. To evaluate this issue, we conducted a set of follow-up analyses of the model's performance. We first asked whether the ordering of the inputs on the similarity dimension was critical to the results. Recall that the shape-matching test object was closer to the exemplar for both the rigid and deformable input sets. This configuration implies that the shape-matching test object is more similar to the material-matching one in the representational state created when children view these stimuli in these tasks. To test the necessity of this similarity relationship for the obtained results, we set up inputs in which the material-matching test object was more similar to the exemplar than the shapematching test object in the deformable stimulus set. Even though this set of inputs was otherwise quite similar to the final set, we were not able to capture the full pattern of results seen in Figure 7 with inputs structured this way.

More specifically, we were only able to adequately capture data from one task at a time with the deformable inputs structured in this way. In order to capture children's yeses to the deformable shape-matching test object in the yes/ no task, the shape-matching test object must overlap somewhat with the exemplar. However, this configuration causes the material-matching test object to be so close to the exemplar that the model chooses it more than the children did in the forced-choice task. This is the critical trade-off seen in children's performance in these tasks - they are sensitive enough to importance of shape for deformable things that they do generalize novel names for deformable things by shape in the yes/no task. However, when forced to choose between shape- and material-matching test objects, they generalize novel names by material similarity more often. This trade-off is realized in the parameters for the deformable set-the shape-matching test object is necessarily closer to the exemplar, resulting in "yes" responses in the yes/no task, but the material-matching test object is stronger, resulting in its greater selection in the forced-choice task. 



Figure 7. Mean proportion of times the model (white bars) selected the shape-matching test object in the forced-choice task as well as the mean proportion of yes responses to the shape and material-matching test objects in the yes/no task for the deformable and rigid stimulus sets. The corresponding data from children in the experiment are also presented (black bars).

There is another element of children's responses, however, that the model is also able to capture - the fact that children's responding is variable. In particular, children do not always pick the material-matching test object with the deformable stimuli in the forced-choice task, and do not always say "yes" to the shape-matching test object in the yes/ no task. Our use of noisy inputs (see Appendix) is critical to this result. This noise model implies that the representational states created when children view these stimuli in these tasks are somewhat variable, although, importantly, this noise is coherent across individual inputs. This means that in the highly-selective forced-choice task, for instance, the (typically) weaker test object may be chosen if noise boosts it's activation above the level of the (typically) stronger input. At the same time, noisy inputs mean that sometimes test objects that are relatively close to the exemplar are weakened such that they cannot support the formation of a stable peak and a "yes" response. Thus, the fit seen in Figure 7 only emerges when the right set of inputs are given to a model with just the right interactive dynamics. This fact serves to further validate the relative positioning of the test objects and exemplar shown in Figure 6, and highlights the importance of the interaction between the specific inputs and the task dynamics in creating behavior.

One final issue with respect to the modeling work presented thus far is the relation between the different interaction functions used to model the two tasks and the participants in our experimental task. In particular, do differences in the interactive dynamics used to capture performance in the two tasks correspond best to different sets of processes in two different children, or can they be seen as task-related differences that emerge on-line in an individual child engaged in a specific task? A close examination of the five interaction parameters that changed across the two tasks suggests the latter. In particular, these parameters may be best viewed as arising from a real-time attentional modulation of the neural circuits responsible for decision making in these two tasks. For example, the large value of the input gain pa- 
rameter, $\kappa$, in the forced-choice task can be thought of as an attentional boost related to the amount of stimulation provided by having multiple inputs simultaneously present. Prior work has demonstrated such changes can occur via attentional systems in real-time in individual participants (Desimone \& Duncan, 1995).

The task differences in the initial resting level, $h_{i}$, and the resting level boost during the response, $h_{b}$ can be viewed in a similar way. The parameter, $h$, is a constant added equally to all locations in the field. Thus, rather than specifying where a peak should form, this parameter modulates the possibility that a peak will form by moving the decision field closer to the peak-formation threshold. Conceptually, the shift in $h$ during the trial reflects the boost in children's attention to the test objects as the tray is slid forward in the forced-choice task or the children are asked to give a response in the yes/ no task. Note that from this perspective, the smaller boost in the yes/no task corresponds to the fact that the stimuli are never moved within reach of the child.

The between-task differences in the final two parameters, interaction inhibition, $w_{i^{\prime}}$ and interaction strength, $w_{e^{\prime}}$ are captured in Figure 5. As can be seen in the figure, the interaction functions for the two tasks are actually very similar, but the function for the forced-choice task has been lowered and stretched. The lowering is due to the higher inhibition value used in the forced-choice task. The stretching of the forcedchoice function is due to the greater interaction strength used in this task, which creates a greater degree of interactivity overall. Importantly, both of these changes can be produced in a more neurally-realistic dynamic field model with separate excitatory and inhibitory layers by changing the resting levels $(h)$ of both layers (Schutte and Spencer, in press; Spencer et al., 2007). Because these changes can be created through the modulation of global resting levels, they can also be thought of as arising via an attentional modulation of the neural populations involved in decision making in these tasks.

\section{Quantitative modeling of developmental changes}

Our dynamic field model captures important differences in the processes that underlie performance in forcedchoice and yes/no novel noun generalization tasks. The excellent fit of the model to the data from 2.5-year-old children in these tasks confirms that the interaction of the input and task dynamics can result in different patterns of noun generalization when the same knowledge representations are processed in two different tasks. Thus, the experimental and simulation results thus far support the perspective that knowledge cannot be separated from the processes that bring it to bear in a task, and helps explain why what children appear to know can look different from task to task. In this section, we extend the modeling work further by exploring another important finding in the literature on children's novel noun generalization-developmental changes in noun generalization from 2 to 4 years of age. We focus on one recent set of experiments that demonstrate changes in noun generalization in a yes/no task.
Samuelson et al. (2008) examined novel noun generalization in 2-, 3-, and 4-year-old children using the same rigid and deformable stimuli used in Experiment 1. The data are shown in Figure 8 (black bars) along with the data from 2.5-year-old children in the current paper. As is clear in the figure, these data suggest differences in the proportion of "yes" responses across rigid and deformable stimulus sets, shape- and material-matching test objects, and age. Our modeling work thus far has captured differences in the amount of shape responding for rigid and deformable sets in the yes/no task, as well as differences in the proportion of yes responses to shape- and material-matching test objects. Our question, then, was whether the DF model could capture developmental differences in responding in the yes/ no task as well.

Three developmental effects stand out in Figure 8. First, the proportion of yes responses to test objects that match rigid exemplars in shape increases from 2 to 3 years of age and remains high (see A in Figure 8). Second, the proportion of yes responses to test objects that match both rigid and deformable exemplars in material drops over this period of development (see Bs in Figure 8). Finally, 3-year-old children demonstrate a higher rate of yes responses to test objects that match deformable exemplars in shape ( $\mathrm{C}$ in Figure 8; see also Samuelson \& Smith, 2000a), compared to 2- and 4 -year-old children (see Samuelson et al. (2008) for statistical analysis and replication of the high shape responding in 3-year-old children).

To capture these developmental differences in the model, we instantiated two hypotheses about changes over development in how children actively represent stimuli in a naming context captured by the input array: (1) that the association between shape and naming increases over development and (2) that stimulus representations become less noisy (i.e., more stable) over development. The first hypothesis fits with a number of studies in the literature on early word learning that suggest children's attention to shape when naming novel objects becomes stronger over development (Gershkoff-Stowe and Smith, 2004; Landau et al., 1988; Samuelson and Smith, 1999; Smith et al., 1992). The second hypothesis fits with recent arguments concerning the nature of developmental change and work using similar DF models (Schöner \& Dineva, 2007). For example, Schutte and Spencer (in press) captured the pattern of developmental change in young children's spatial memory via changes in the specifics of the local excitation/lateral inhibition function in a DF model of spatial cognition. These changes in neural interaction resulted in more stable representations of items in working memory, that is, reduced noise in how children actively represented stimuli as hypothesized here. The critical question is whether the complex pattern of results would emerge when these two developmental changes to children's representations interact with the real-time decision processes captured in the model.

\subsection{Methods}

The model was identical to that used previously except where noted below. That is, we used the same interactive 

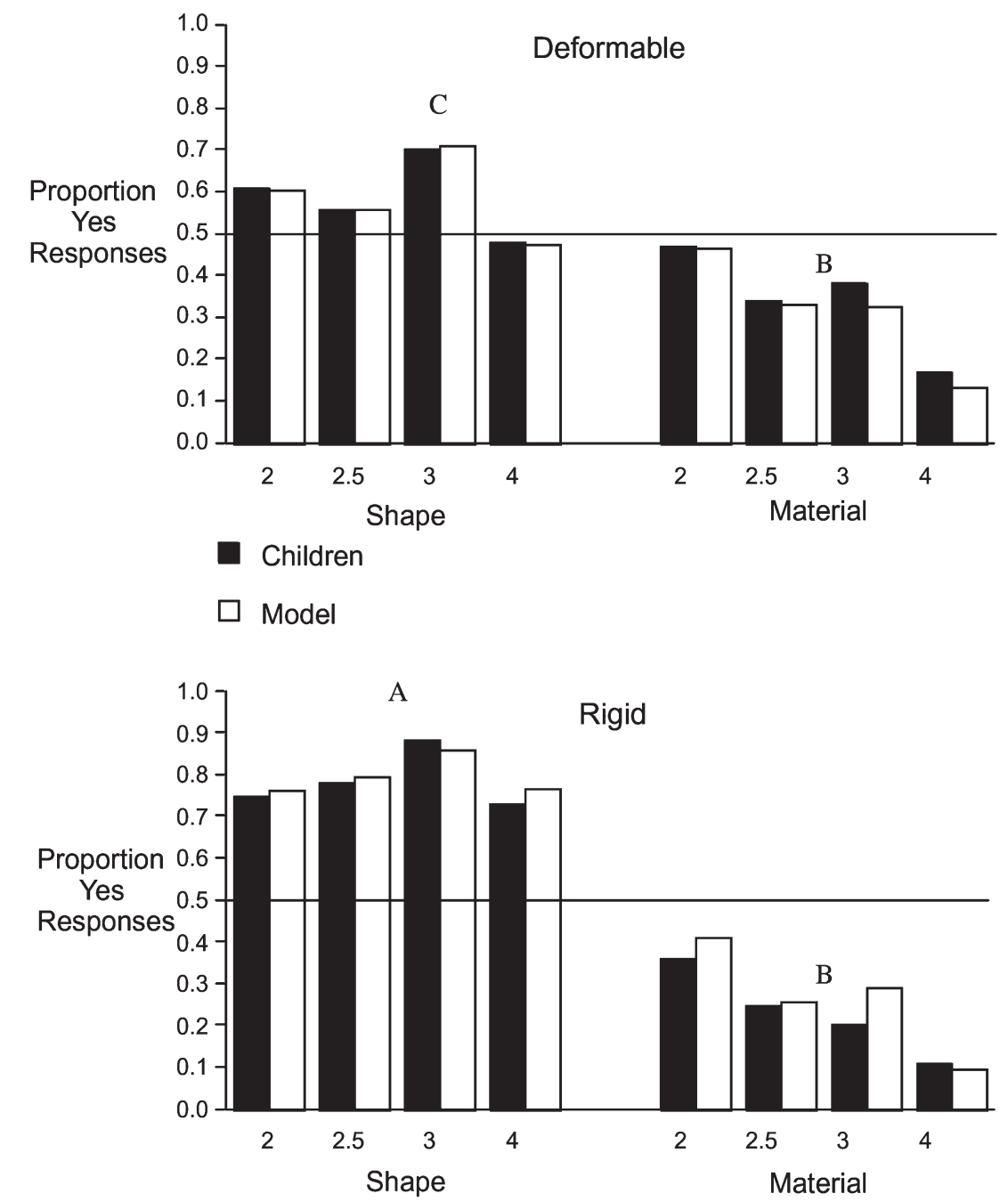

Figure 8. Mean proportion of yes responses by 2-, 2.5-, 3-, and 4-year-old children (black bars), and the DF model (white bars) to shape- and material-matching test objects in the yes/no task for deformable and rigid stimulus sets. Thirty-two month-old data are from the current paper. Two-, 3- and 4-year-old data are from Samuelson et al. (2008).

dynamics parameters for the yes/no task and the same input parameters for the rigid and deformable stimuli. To simulate development (2-, 3-, and 4-year-old children), we scaled the noise strength, $q$, and varied the strength of the shape inputs relative to the values used previously for our simulations of the data from 2.5-year-old children. Specifically, noise strength $(q)$ decreased systematically over development, while the strength of the shape inputs increased over development. The differences in noise and shape strength relative to the prior simulations are depicted in Figure 9.

Simulations were conducted in MATLAB (Mathworks Inc.) on a PowerMac G5 with dual $1.8 \mathrm{GHz}$ processors. Otherwise, the simulations were identical in detail to the previous set. Each run of the model simulated a single trial with a given stimulus set. We conducted 200 repetitions with each stimulus set (deformable and rigid) at each developmental level, and verified that the model converged on similar values across 10 sets of 400 simulations (200 repetitions $\times 2$ stimulus sets) at each developmental level.

\subsection{Results and discussion}

We calculated the mean number of times the model made "yes" and "no" responses for the deformable and rigid stimulus sets at each developmental level. Data from the model simulations are presented in Figure 8 (white bars). As is clear in the figure, the model fit the data very well. To quantify the fit, we calculated the root mean squared error across the two stimulus sets and three developmental levels. This value was .04 for the pictured simulation. We also examined the average RMS for the 10 sets of 400 simulations we conducted at each age level. The average was .05 with a standard deviation of .014 and a range of .03-.08. Thus, the model's performance shown in Figure 8 was quite robust.

These simulations, then, suggest that changes in young children's attention to shape and material from 2 to 4 years of age are related to changes in the strength of the link between naming tasks and attention to shape, and the precision of the representational states created when children view the stimuli in these tasks. Both of these developmen- 


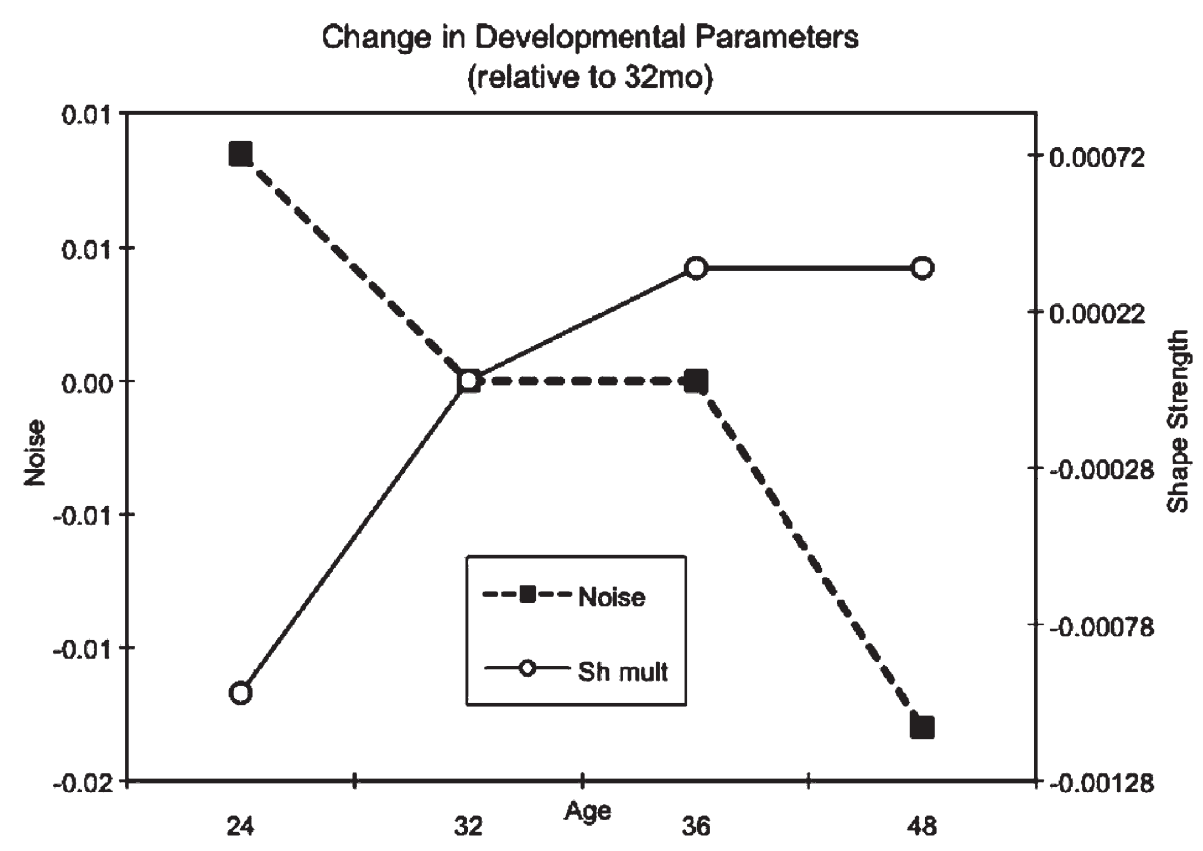

Figure 9. Change in noise strength $(q)$ and shape strength parameters across simulated developmental levels relative to simulations of 2.5-year-old children.

tal hypotheses have support in the literature. Nevertheless, an important question is whether both of these changes are necessary to create the observed pattern in the data. To examine this issue, we attempted to capture the pattern of results seen in Figure 8 in two additional sets of simulations that included changes to either noise strength or shape input strength separately. In the simulations that manipulated noise strength only, the best parameter values were .05 , .045 , and .038 for the 2-, 3-, and 4-year-old simulations, respectively. These values resulted in a average RMS of .05 for the best single run at each developmental level, but an overall average RMS of .078 (SD = .039, range .02-.14) across 10 sets of 400 simulations at each developmental level. A two sample $t$-test revealed that the RMS values for the noiseonly simulations were significantly poorer than those for the main simulations reported above, $t(58)=-3.65, p<.001$. This poor performance is due to the fact that reducing noise in the model serves to reduce the number of "yes" responses overall, which is not the developmental pattern children show between 2 and 4 years of age. Four-year-old children do show an overall reduction in yes responses compared to 3-year-old children. However, between 2 and 3 years of age, the number of yes responses increases for shape-matching test objects while decreasing for material-matching test objects (compare A and B in Figure 8). Thus, while changing noise strength alone results in relatively low RMS values, there is no consistent set of changes to this parameter alone that can simultaneously capture children's responding to the shape- and material-matching test objects.

A similar pattern emerged in the simulations that manipulated shape input strength only. The best parameter values for this set of simulations were 1.00000001, 1.00036, and .9995 for the 2-, 3-, and 4-year-old simulations, respectively. Because the difference in 2.5- and 3-year-old children's re- sponding was captured in the main simulations with a change in the noise strength parameter alone, we used the main simulations for 2.5-year-olds in this follow-up. These values resulted in a average RMS of .08 for the best single run at each developmental level, and an overall average RMS of .088 (SD = .026, range .04-.13) across 10 sets of 400 simulations at each developmental level. A two sample $t$-test revealed that the RMS values for the shape-multiplier-only simulations were significantly poorer than those for the main simulations reported above, $t(58)=-6.87, p<.001$. This poor performance is due to the fact that increasing the strength of the shape input does not influence responding to the material-matching stimuli which contrasts with results shown in Figure 8. Thus, while changing shape strength alone results in relatively low RMS values, it does not capture the full pattern of results. In particular, there are no consistent set of changes to the shape input strength alone that can capture both the increase the number of yeses to test objects that match exemplars in shape and the decrease in the number of yeses to test objects that match exemplars in material that children show over development.

Overall, then, these simulations suggest that our dynamic field model can capture important developmental differences in children's noun generalization from 2 to 4 years of age with relatively modest and conceptually appealing changes to two parameters. These parameters reflect the strength of attention to shape in naming tasks and the amount of noise in the representations children form when viewing the stimuli in a naming context. Thus, these changes correspond to prior hypotheses concerning the nature of developmental change in novel noun generalization (Jones and Smith, 1993; Samuelson, 2002; Smith, 2001; Smith and Samuelson, 2006; Smith et al., 2002) and the stability of children's representations (Schöner \& Dineva, 2007). Together with 
the prior simulations of performance differences in forcedchoice and yes/no tasks, the DFT provides novel insights into how knowledge is brought to bear in a moment and how that knowledge changes over development.

\section{General discussion}

The goal of this paper was to examine the processes that bring young children's knowledge of nominal categories to bear as they generalize novel nouns. As in prior studies, we found that children robustly generalize novel names for rigid objects by similarity in shape, and that this bias appears in both forced-choice and yes/no novel noun generalization tasks (Booth et al., 2005; Landau et al., 1988). Nevertheless, we found that children generalized names for deformable things by material in a forced-choice task, but not in the yes/no task, even though the children's age and vocabulary size were equated across tasks, and all children saw the same stimuli and demonstrations highlighting the material of deformable exemplars. This task-dependent performance when generalizing names for deformable things fits both with prior studies of noun generalization (Gathercole \& Min, 1997; Samuelson et al., 2007; Samuelson \& Smith, 2000a), and adult judgments of the similarity structure of categories of deformable things (Samuelson and Smith, 1999; Samuelson and Smith, 2000a). The current work makes a contribution by showing how the interaction of represented knowledge and task processes can result in consistent performance across tasks in some cases (rigid stimuli) and variable performance in others (deformable stimuli).

A dynamic field model that captured critical differences in the decision processes in the forced-choice and yes/no tasks provided a demonstration that processing differences can yield behavioral differences between tasks even when the same vocabulary knowledge is accessed. This model also provided an excellent quantitative fit to the experimental data from both the rigid and deformable stimulus sets. Further, the model was able to capture developmental changes in children's performance in the yes/no task. In this way, then, the model provides a window onto both the real-time processes that can produce different patterns of responses in different task contexts, and what changes developmentally to produce different performance at different ages (see also Plumert, 2008).

\subsection{Real-time processes}

The DF model presented here is the first processed-based model of the real-time noun generalization behaviors that form the basis of current theories of early word learning. As such, one key contribution of our DF model of children's naming behaviors comes from the real-time specification of the processes that bring children's knowledge of nominal categories to bear in different noun generalization tasks. Studies of the early noun vocabulary have documented a clear and systematic association between nominal categories of rigid things and the importance of shape for category organization (Samuelson, 2000; Samuelson and Smith, 1999;
Samuelson et al., 2008). These studies also suggest a more complex relationship between nominal categories of deformable things and the importance of shape and/or material for category organization. We contend that the more complex relationship between category organization and naming for deformable things allows signatures of real-time processes to be seen in children's noun generalizations - children generalize names for deformable things by shape in yes/no tasks, but by material in forced-choice tasks (see also Gathercole \& Min, 1997; Samuelson et al., 2007; Samuelson \& Smith, 2000a). Our DF model suggests that the key processing difference reflected in these behavioral data is grounded in the competitive nature of the forced-choice task that allows for direct comparison of the test objects and asks the child to pick one object relative to the yes/no task that does not. In the DF model, decisions in the forced-choice task are based on which input peak wins the competition and suppresses other competitors to enter a self-sustaining state. In contrast, decisions in the yes/no task are based on the similarity of each individual test object input to the exemplar; if the input is similar enough it will blend with the exemplar's input in the decision field and will create a self-sustaining peak (a "yes" response). The excellent fit of the simulations presented here to children's data support our proposal regarding the differing processes at work in the two tasks. In addition, the simulations support the proposals that attention to material is slightly stronger for categories of deformable things, resulting in generalization by material in the forcedchoice task, but that things that match in shape are more similar overall, resulting in generalization by shape in the yes/no task.

This modeling work suggests that further exploration of these real-time processes could help clarify some of the discrepancies in the literature on early noun learning. For example, Gershkoff-Stowe and Smith (2004) found that it was not until children had 50 nouns in their productive vocabulary that they began to demonstrate a shape bias with rigid stimuli. In contrast, Booth et al. (2005) found that 18-montholds with an average of 19 count nouns in their productive vocabulary generalized novel names for novel rigid objects by shape. The work presented here suggests that these differences may reflect the importance of real-time comparison processes in determining how children's accumulated knowledge of nominal categories manifest in the particulars of young children's noun generalizations. GershkoffStowe and Smith (2004) used a five-item forced-choice task. In contrast, Booth et al. (2005) used a three-item forcedchoice task that included multiple named exemplars and vignettes describing the exemplars as artifacts. More test objects means that children have more items to attend to and more comparisons to make when generalizing a novel name. In the DFT, the presentation of multiple choice items increases competition among the choices. This would reduce the influence of the small differences in the strength of inputs to the model that were critical to capturing the current data, and would thus lead to more chance responding by the model (for evidence that the number of choices influences behavior in the DFT see Spencer et al. (2006)). This suggests then, that children may be able to demonstrate a 
reliable shape bias based on weaker underlying representations of the stimuli in a three-item forced-choice task compared to a five-item forced-choice task. Likewise, the supports Booth et al. (2005) added to their task (e.g., presenting multiple exemplars and a contrast item, and naming exemplars multiple times in vignettes) may have also helped younger children make decisions based on weaker underlying knowledge.

Thus, this modeling work serves as a reminder that when children acquire 50 nouns, it is not the case that a switch flips in their head turning on a shape bias. Rather, the behavior we call the shape bias is the emergent product of children's accumulating knowledge of how things are named and the real-time processes of the task (Samuelson \& Smith, 2000b). As children acquire more and more words, they learn more and more about the regularities inherent in the language they are learning. For children learning English, one of these regularities is that many count nouns refer to solid things in categories organized by similarity in shape (Gathercole and Min, 1997; Samuelson and Smith, 1999). This accumulating knowledge can serve as the basis of novel noun generalizations and subsequent word learning as captured by the Attentional Learning Account proposed by Smith and colleagues to explain the development and realization of the shape bias (Smith, 2000; Smith and Samuelson, 2006; Yoshida and Smith, 2003). What the current work adds to this is a demonstration of the importance of the real-time processes in making that connection between the child's prior word learning history and the hereand-now of a novel word in a novel situation with novel stimuli. In this sense, our model highlights that differences in the specific results of individual studies are not problems, but rather, important windows onto the underlying real-time processes that have received relatively little attention in work on early word learning.

Another key contribution of the model in this context is that it is, to our knowledge, the first real-time process model of the comparison processes in word learning tasks (for other process models of comparison see, e.g. Goldstone, 1994; Larkey and Love, 2003). Based on studies in infant categorization, early word learning, and the development of analogical reasoning, a number of authors have argued recently for the importance of comparison in cognitive processing and early word learning (Gentner and Medina, 1998; Gentner and Namy, 2006; Namy and Gentner, 2004; Oakes and Madole, 2003; Sandhofer, 2001). However, the nature of the comparison process in this literature has been underspecified. The model presented here takes a first step towards greater specification by detailing the nature of the comparison process - interaction of a set of neurons according to a local excitation/lateral inhibition function - as well as the result-a stable peak of activation at a particular location on an ordered dimension. Further, the modeling work presented here explicitly ties behavior in novel noun generalization tasks to a more general theory of decision processes and a theoretical framework - the DFT - that is grounded in neural principles (see Simmering, Schutte, \& Spencer, 2008; Spencer, Dineva, \& Schöner, in press; Spencer et al., 2007). Thus, our comparison process is neurally plausible.
These contributions notwithstanding, it will be important in the future development of this model to incorporate a more rigorous representation of similarity. The model presented here used a very basic notion of similarity - a single dimension where distance along that dimension captures overall similarity - akin to the use of distance metrics in other models (e.g., Kruschke, 1992; Nosofsky, 1987; Samuelson, 2002). Although the similarity structure of our inputs was well grounded in the literature, a more complete model should include separate dimensions for particular feature dimensions such as shape, material, size, color, and so on. Adding a more complex representation of different object properties would enable fine-grained distinctions between the relative importance of features such as rounded corners and straight edges for rigid vs. nonrigid or nonsolid things. Further, such a representation would allow for easier manipulation of the relative importance of such features with changes in the nominal categories in the productive vocabulary.

Towards this end, Faubel and Schöner (2008) have used coupled, multi-dimensional dynamic fields in an autonomous robot that quickly learned to name 30 objects (see also Johnson et al., 2008). Importantly, this work suggests that the similarity structure of nominal categories - that is, the fact that things called "banana" are more similar to each other and to things called "squash" than they are to things called "stapler" - is emergent from the system's prior history of naming, and correlations between particular names and activation of relevant feature dimensions in a given task context. Incorporating a more complex representation of similarity in our model will also require, however, corresponding advances in our understanding of how children represent similarity. This has been limited in the past by procedural constraints such as the small number of trials an individual child can perform in a single experimental session. We are currently working on a new method for eliciting similarity judgments in children that will overcome some of these limitations.

\subsection{Toward developmental process}

The fact that we were able to use the same model to capture differences in children's naming of deformable and rigid things from 2 to 4 years of age suggests that understanding processes at the level of individual trials can aid in understanding developmental change. The developmental simulations presented here point to two critical changes in knowledge representations over this age range: (1) an increase in the strength of the association between naming tasks and shape similarity and (2) a reduction in the amount of noise in the representational states created when young children view stimuli in these tasks. These relatively simple developmental changes are well supported in the literature. The critical insight from the model is that the complex pattern of responding we see across this age range emerges when these two simple, quantitative and systematic developmental changes are combined and brought to bear in a moment.

Further understanding of the complexities of performance in these tasks and over development will come from 
future work that "closes the loop" between real-time performance and development. We need to understand how individual behaviors on the real-time scale accumulate to create the differences in performance seen in children of different ages and vocabulary levels. According to the Attentional Learning Account proposed by Smith and colleagues (Smith, 2001; Smith and Samuelson, 2006), biases such as the shape bias are learned products of the child's experiences with language. Thus, the very early word learning experiences of the child influence future word learning via accumulation of regularities over multiple individual exposures to words and categories. Importantly, the DFT provides a process by which individual experiences can build upon each other to influence the dynamics of future real-time behavior and thereby create biases. Specifically, in their work on spatial cognition, Spencer and colleagues (Spencer et al., 2007) have used decision fields, similar to the ones used here, coupled to long-term memory fields (see also Faubel \& Schöner, 2008). In these models, self-sustaining working memory peaks generated over the course of individual trials leave activation traces in long-term memory that can feed back and influence working memory (implementing a form of Hebbian learning; for discussion, see Spencer et al., in press). This system, then, provides a concrete process by which statistics of past decisions can accumulate over multiple individual trials and, in turn, can be brought to bear on current behavior.

These neurally grounded models have been used to capture changes on the intermediate timescale of biases that accumulate over the course of an experiment as well as differences in children's spatial memory abilities from 6 months to 6 years of age (see Spencer et al. (2007) for a review). For example, Hund and Spencer (2003) found that if adult participants are asked to recall three locations but one location is responded to more often than the others, memory responses come to be biased towards the more frequently responded to-location. Similarly, Divena and colleagues (Dineva, 2005) have found that infants' trial-by-trial responses in the classic Piagetian A-not-B task impact their subsequent performance. In fact, infants who early on in the task spontaneously reach to what will be the correct location later, are less likely to make search errors on the critical test trials. In the DF model used to capture this finding, it is the individual decisions made on each trial that are the critical influence on long-term memory and thus subsequent behavior. We have found a similar behavioral result in recent work on 24-month-old children's generalizations of names for nonsolid substances. As reported in Samuelson and Horst (2007), these young children become less likely to generalize a novel name for a novel solid rigid object by shape if, over the course of the experimental session, they have previously seen many nonsolid substances and solid things broken into pieces. A critical question will be whether this trialby-trial effect, like those found in previous reports that tap spatial and sensorimotor systems, is necessarily based on the naming decisions children make over the course of the experiment. Answering this question will have clear implications for the nature of the process by which vocabulary statistics accumulate to create biases. More generally, incorporating a coupled long-term memory system into the current model will provide a means to further explore how the development of word learning biases might arise from individual naming experiences.

\subsection{Conclusions}

The goal of the current work was to examine an issue at the core of modern developmental science and cognitive psychology: is it useful to think of knowledge and process as separate or must we move towards a view in which knowledge is understood in conjunction with, and relative to, the processes that bring it to bear in a moment in a task. Our work is clearly inspired by this second perspective which acknowledges that our only window onto knowledge is via the behaviors we study and thus necessarily through the pane of the processes that create those behaviors. This is clearly illustrated in the data from our first experiment in which the influence of real-time process on "knowledge" is seen in the differing generalizations of children equal in age and vocabulary knowledge when doing different tasks. It is also clearly seen in the modeling work presented here which demonstrates the dynamic interaction of represented knowledge and decision processes in creating the patterns of children's behavior seen in specific tasks and over development. And because we used an authentic process model that generated individual responses on individual trials just as children do (rather than mapping simulated activation levels onto response probabilities) the DF model presented here demonstrates the critical nature of real-time processes in children's individual naming behaviors.

In this respect, this work follows the lead of pioneers such as Gibson (1969) and Thelen and Smith (1994) in examining how far the real-time processes of the system can take us in understanding behavior and behavioral development. Note, however, that this is not to say that the underlying knowledge must be as limited as the representations used in the current work. Rather, the point is that far less may be required from underlying knowledge than might have been expected because the dynamic interaction of that knowledge in a task in real-time is so critical in shaping behavior. Additionally, the developmental simulations presented here suggest the importance of understanding these real-time behaviors when considering the nature of developmental change. Thus, the model points to important generalizations that can be made about the role of comparison, stimulus similarity, noise, and acquired knowledge in directing children's behavior at a given point in development. However, this work also makes clear the necessity of future work examining individual differences for a full understanding of knowledge, behavior, and process in word learning and cognitive development more generally.

Future expansion of the model within the more general DF framework offers the additional possibility of bridging the gap between the real-time performance seen in our experiment, and captured in our task simulations, and the long-term changes captured in our developmental simulations. In particular, because this model is inspired by a line of theoretical, computational, and empirical work examining 
a range of behaviors from spatial memory, to change detection, habituation, discrimination and spatial language, it ties children's noun generalization behaviors to this larger picture of cognitive process. In this way, the current model provides a means to generalize insights about cognition gained in relatively simple sensorimotor examples (like the A-not-B task) to tasks that tap higher-level cognition, categories, and linguistic systems. Towards this end, the current data and model demonstrate the importance of considering behavior in a task, and support a view of knowledge as created in the moment from the interaction of the child's history and realtime, task-specific dynamics.

\section{Acknowledgments}

Preparation of this manuscript was supported by NICHD Grant No. 5 R01 HD045713 to the first author. Portions of the data were presented at the 2006 meeting of the Cognitive Science Society. The authors wish to thank John Spencer, Jodie Plumert, and Linda Smith for thoughtful discussions of this work and helpful comments on earlier versions of the manuscript. We also thank Ryan Brink, Brandi Dobbertin, and the members of the Language and Category Development Lab for assistance with data collection. Finally we thank the parents and children who participated in the studies, without whom this work would not be possible. Jessica Horst is now at the Department of Psychology, University of Sussex, UK.

\section{Appendix.}

The Dynamic Field Theory is in a class of bi-stable neural networks where "on" and "off" states coexist (Amari and Arbib, 1977; Erlhagen and Schöner, 2002; Thelen et al., 2001). Thus, these networks can capture stable decisionsthe "on" state-as well as cases where stable decisions are not reached - the "off" state. The continuous evolution of activation in the decision fields shown in Figure 4 is specified by a differential equation that determines a rate of change, $d u(x, t)$ / $d t$, for every activation level, $u(x, t)$, at every field location, $x$, and any moment in time, $t$. The basic stabilization mechanism of the field is modeled by an inverse relationship between the rate of change and the current level of activation, $u(x, t)$. This means that at high levels of activation, negative rates of change drive activation down, while at low levels, positive rates of change drive activation up. The activation level that emerges is a function of the balance of different inputs and interactions in the field. For example, when a negative resting level, $h<0$, coexists with a source of excitatory input, $S>0$, then the resulting stable state of the activation dynamics:

$$
\tau d u(x, t) / d t=-u(x, t)+h+S(x, t)
$$

is $u(x, t)=h+S(x, t)$, the level at which positive and negative rates of change balance so that $d u / d t=0$. Note that $\tau$, a parameter that fixes the time scale of the activation field, was set to $75 .^{2}$

2. We assume that one time step is roughly equivalent to $4 \mathrm{~ms}$, thus making the time between stimulus onset and response request about $2.5 \mathrm{~s}$, and thus giving the model $2 \mathrm{~s}$ to respond (see Section 3.2.2). One advantage of process-based models such as this one is the ability to make predictions about reaction time. Unfortunately, however, standard procedures for these tasks are not controlled enough to obtain reaction time measurements in the young children studied here. We are currently pursuing the development of procedures that would overcome this limitation.
When the rate of change of activation at a field site, $x$, depends not only on the activation level, $u(x, t)$, and current inputs, $S(x, t)$, but also on the activation levels, $u\left(x^{\prime}, t\right)$, at other field sites, $x^{\prime}$, then activation dynamics are interactive. Locally excitatory/laterally inhibitory interactions are described by an interaction function, $w\left(x-x^{\prime}\right)$, such that

$$
w\left(x-x^{\prime}\right)=\frac{w_{e}}{\sqrt{2 \pi}}\left[\exp \left(-\frac{\left(x-x^{\prime}\right)^{2}}{2 \sigma_{w}^{2}}\right)\right]-w_{i}
$$

In the reported simulations, this interaction $\sigma_{\mathrm{w}}$ was always set to 12.0. Only sufficiently activated sites, $x^{\prime}$, contribute to the interaction. This is expressed by passing activation through a sigmoidal function:

$$
f\left(u\left(x^{\prime}, t\right)\right)=1 /\left(1+\exp \left(-\beta u\left(x^{\prime}, t\right)\right)\right)
$$

Such threshold functions are necessarily nonlinear and are the basis for bi-stability. For the simulations reported here $\beta$ was set to 5.0. This creates a relatively sharp threshold function and thus allows for the strong nonlinearities needed to create stable activation peaks (i.e., stable decisions).

Thus, the full model took the form:

$$
\tau d u(x, t) / d t=-u(x, t)+h+\kappa S(x, t)+\int d x^{\prime} w\left(x-x^{\prime}\right) f\left(u\left(x^{\prime}, t\right)\right)
$$

where $\kappa$ was a general gain factor that modulated the strength of the input, $S(x, t)$.

Inputs to the model took the form of Gaussians represented by the following equation:

$$
S(x, t)=A \exp \left[-\frac{\left(x-x_{c}\right)^{2}}{2 \sigma^{2}}\right]
$$

where $A$ is the amplitude or strength of the input, $x_{c}$ is the central position of the input along the similarity dimension, and $\sigma$ determines the precision of the input. The specific inputs to the model varied depending on the task and the stimulus set. For the forced-choice task, the exemplar and both the shapematching and material-matching test objects were all presented at once. Thus, the input equation for this task was:

$$
S_{F C}(x, t)=S_{\text {Exemplar }}(x, t)+S_{\text {Shape }}(x, t)+S_{\text {Material }}(x, t)
$$

In the yes/no task, each test object was presented individually with the exemplar. Thus, for one trial of this task the equation was:

$$
S_{Y N \_1}(x, t)=S_{\text {Exemplar }}(x, t)+S_{\text {Shape }}(x, t)
$$

and for the other it was:

$$
S_{Y N \_2}(x, t)=S_{\text {Exemplar }}(x, t)+S_{\text {Material }}(x, t)
$$

The stimuli for the deformable and rigid sets varied in the strength, precision, and location of the test objects (the exemplar was always in the same location). Details of these stimulus sets are discussed below.

Noise was added to the model using fluctuating amplitudes for all inputs. This introduces a form of spatially correlated noise that is consistent with neural principles (Dineva, 2005; Schutte et al., 2003). Thus, the generic equation for the inputs including noise was:

$$
S(x, t)=A+q \xi(\mathrm{t})) \exp \left[-\frac{\left(x-x_{c}\right)^{2}}{2 \sigma^{2}}\right]
$$


The noise strength, $q$, was set to .05 for all simulations. $\xi$ was a normally distributed random number. Different random numbers were selected at each time step and for each component of the input (e.g., shape-matching test object vs. material-matching test object) independently.

\section{References}

Amari and Arbib, $1977 \varangle$ S. Amari and M. A. Arbib, Competition and cooperation in neural nets. In: J. Metzler, Editor, Systems neuroscience, Academic Press (1977).

Barsalou, 1999 L. W. Barsalou, Perceptual symbol systems, Behavioral and Brain Sciences 22 (1999), pp. 577-600.

Bastian et al., $1998 \triangleleft$ A. Bastian, A. Riehle, W. Erlhagen, and G. Schöner, Prior information preshapes the population representation of movement direction in motor cortex, NeuroReport 9 (1998), pp. 315-319.

Bastian et al., $2003<$ A. Bastian, G. Schöner, and A. Riehle, Preshaping and continuous evolution of motor cortical representations during movement preparation, European Journal of Neuroscience 18 (2003), pp. 2047-2058.

Berthier et al., $2000<$ N. E. Berthier, S. DeBlois, C. R. Poirier, J. A. Novak, and R. K. Clifton, Where's the ball? Two- and three-year-olds reason about unseen events, Developmental Psychology 36 (2000), pp. 394-401.

Bloom, 20004 P. Bloom, How children learn the meanings of words, The MIT Press, Cambridge, MA (2000).

Bloom and Markson, $1998<$ P. Bloom and L. Markson, Intention and analogy in children's naming of pictorial representations, Psychological Science 9 (3) (1998), pp. 200-204.

Booth and Waxman, 20024 A. E. Booth and S. R. Waxman, Word learning is 'smart': Evidence that conceptual information affects preschoolers' extension of novel words, Cognition 84 (2002), pp. B11-B22.

Booth et al., 20054 A. E. Booth, S. R. Waxman, and Y. T. Huang, Conceptual information permeates word learning in infancy, Developmental Psychology 41 (3) (2005), pp. 491-505.

Clearfield et al., $2006 \varangle$ M. W. Clearfield, F. J. Diedrich, L. B. Smith, and E. Thelen, Young infants reach correctly in A-not-B tasks: On the development of stability and perseveration, Infant Behavior and Development 29 (2006), pp. 435-444.

Colunga and Smith, $2008-$ E. Colunga and L. B. Smith, Knowledge embedded in process: The self-organization of skilled noun learning, Developmental Science 11 (2008), pp. 195-203.

Desimone and Duncan, $1995<\mathrm{R}$. Desimone and J. Duncan, Neural mechanisms of selective visual attention, Annual Review of Neuroscience 18 (1995), pp. 193-222.

Diedrich et al., $2001<$ F. J. Diedrich, T. Highlands, E. Thelen, and L. B. Smith, The role of target distinctiveness in infant perseverative reaching errors, Journal of Experimental Child Psychology 78 (2001), pp. 263-290.

Diedrich et al., $2000<$ F. J. Diedrich, E. Thelen, L. B. Smith, and D. Corbetta, Motor memory is a factor in infant perseverative error, Developmental Science 3 (2000), pp. 479-494.

Diesendruck and Bloom, $2003<$ G. Diesendruck and P. Bloom, How specific is the shape bias?, Child Development 74 (1) (2003), pp. $168-178$.

Dineva, 20054 E. Dineva, Dynamical field theory of infant reaching and its dependence on behavioral history and context, Institue für Neuroinformatik and International Graduate School for Neuroscience, Bochum, Germany (2005).

Durstewitz et al., $2000 \triangleleft$ D. Durstewitz, J. K. Seamans, and T. J. Sejnowski, Neurocomputational models of working memory, Nature 3 (2000), pp. 1184-1191.

Erlhagen and Schöner, $2002 \triangleleft$ W. Erlhagen and G. Schöner, Dynamic field theory of movement preparation, Psychological Review 109 (2002), pp. 545-572.

Faubel and Schöner, $2008 \varangle$ C. Faubel and G. Schöner, Learning to recognize objects on the fly: A neurally based dynamic field approach, Neural Networks 21 (2008), pp. 562-576.

Fenson et al., $1994<$ L. Fenson, P. S. Dale, J. S. Reznick, E. Bates, D. Thal, et al. (1994). Variability in early communicative development, 59(5, Serial No. 5).
Freeman, $2001 \triangleleft$ W. J. Freeman, The behavior-cognition link is well done: The cognition-brain link needs more work, Behavioral and Brain Sciences 24 (2001), pp. 42-43.

Gathercole et al., $1995<$ V. C. Gathercole, L. Cramer, S. C. Somerville, and M. J. o. d. Haar, Ontological categories and function: Acquisition of new names, Cognitive Development 10 (1995), pp. 225-251.

Gathercole and Min, 1997 V. C. Gathercole and H. Min, Word meaning biases or language-specific effects? Evidence from English, Spanish and Korean, First Language 17 (1997), pp. 31-56.

Gathercole and Whitfield, $2001<$ V. C. Gathercole and L. C. Whitfield, Function as a criterion for the extension of new words, Journal of Child Language 28 (2001), pp. 87-125.

Gentner and Medina, $1998<$ D. Gentner and J. Medina, Similarity and the development of rules, Cognition 65 (1998), pp. 263-297.

Gentner and Namy, $2006 \triangleleft$ D. Gentner and L. L. Namy, Analogical processes in language learning, Current Directions in Psychological Science 15 (6) (2006), pp. 297-301.

Gershkoff-Stowe and Smith, $2004<$ L. Gershkoff-Stowe and L. B. Smith, Shape and the first hundred nouns, Child Development 74 (4) (2004), pp. 1098-1114.

Gibson, 19694 E. J. Gibson, Principles of perceptual learning and development, Prentice-Hall, Englewood Cliffs, NJ (1969).

Glenberg et al., 2001 A. M. Glenberg, M. R. Cowart, and M. P. Kaschak, An affordance field for guiding movement and cognition, Behavioral and Brain Sciences 24 (2001), pp. 43-44.

Goldstone, $1994 \varangle$ R. L. Goldstone, Similarity, interactive activation, and mapping, Journal of Experimental Psychology: Learning, Memory, and Cognition 20 (1994), pp. 3-28.

Hund and Spencer, $2003<$ A. M. Hund and J. P. Spencer, Developmental changes in the relative weighting of geometric and experience-dependent location cues, Journal of Cognition and Development 4 (2003), pp. 3-38.

Imai et al., $1994-$ M. Imai, D. Gentner, and N. Uchida, Children's theories of word meaning: The role of shape similarity in early acquisition, Cognitive Development 9 (1) (1994), pp. 45-75.

Jancke et al., $1999 \triangleleft$ D. Jancke, W. Erlhagen, H. R. Dinse, A. C. Akhavan, M. Giese, and A. Steinhage et al., Parametric population representation of retinal location: Neuronal interaction dynamics in cat primary visual cortex, Journal of Neuroscience 19 (1999), pp. 9016-9028.

Johnson et al., $2008<$ J. S. Johnson, J. P. Spencer, and G. Schöner, G. Moving to higher ground: The dynamic field theory and the dynamics of visual cognition. In F. Garzón, A. Laakso, \& T. Gomila (Eds.), Dynamics and psychology. New Ideas in Psychology, 26, 227251 (Special issue; 2008).

Jones and Smith, $1993 \varangle$ S. S. Jones and L. B. Smith, The place of perception in children's concepts, Cognitive Development 8 (1993), pp. 113-139.

Kruschke, $1992 \varangle$ J. K. Kruschke, ALCOVE: An exemplar-based connectionist model of category learning, Psychological Review 99 (1) (1992), pp. 22-44.

Landau et al., $1988 \triangleleft$ B. Landau, L. B. Smith, and S. S. Jones, The importance of shape in early lexical learning, Cognitive Development 3 (1988), pp. 299-321.

Landau et al., 1998 B. Landau, L. B. Smith, and S. S. Jones, Object shape, object function and object name, Journal of Memory and Language 38 (1998), pp. 1-27.

Larkey and Love, 20034 L. B. Larkey and B. C. Love, CAB: Connectionist analogy builder, Cognitive Science 27 (2003), pp. 781-794.

Luce, $1963 \varangle$ R. D. Luce, Detection and recognition. In: R. D. Luce, R. R. Bush and G. Eugene, Editors, Handbook of mathematical psychology, Wiley, New York (1963).

Markman, 2001 A. B. Markman, Are dynamical systems the answer, Behavioral and Brain Sciences 24 (2001), pp. 50-51.

Mix et al., $1996 \varangle$ K. S. Mix, J. Huttenlocher, and S. C. Levine, Do preschool children recognize auditory-visual numerical correspondences?, Child Development 67 (1996), pp. 1592-1608.

Munakata and McClelland, 20034 Y. Munakata and J. L. McClelland, Connectionist models of development, Developmental Science 6 (2003), pp. 413-429.

Namy and Gentner, $2004 \varangle$ L. L. Namy and D. Gentner, The role of comparison in children's early word learning. In: D. G. Hall and S. S. Waxman, Editors, Weaving a lexicon, MIT Press, Cambridge, MA (2004), pp. 533-568. 
Nosofsky, 19874 R. M. Nosofsky, Attention and learning processes in the identification and categorization of integral stimuli, Journal of Experimental Psychology: Learning, Memory, and Cognition 13 (1987), pp. 87-108.

Oakes and Madole, 2003 L. M. Oakes and K. L. Madole, Principles of developmental change in infants' category formation. In: D. H. Rakison and L. M. Oakes, Editors, Early category and concept development: Making sense of the blooming, buzzing confusion, Oxford University Press, Oxford (2003), pp. 132-158.

Plumert, 2008 J. M. Plumert, Children's thinking is not just about what's in the head: Understanding the organism and environment as a unified system. In: R. V. Kail, Editor, Advances in child development and behavior, Academic Press, San Diego, CA (2008), pp. 373-417.

Port and VanGelder, $1995 \checkmark$ R. Port and T. VanGelder, Mind as motion: Explorations in the dynamics of cognition, MIT/Bradford, Cambridge, MA (1995).

Samuelson, 20004 Samuelson, L. K. (2000). Statistical regularities in vocabulary guide language acquisition in 15-20-month-olds and connectionist models. Unpublished Dissertation. Bloomington, Indiana: Indiana University.

Samuelson, 20024 L. K. Samuelson, Statistical regularities in vocabulary guide language acquisition in connectionist models and 15-20-month-olds, Developmental Psychology 38 (6) (2002), pp. 1016-1037.

Samuelson and Bloom, 2008 L. K. Samuelson and P. Bloom, eds. (2008). What counts as an explanation of development: The shape bias as a case study (special section). Developmental Science, 11, $183-222$.

Samuelson and Horst, 2007 L. K. Samuelson and J. S. Horst, Dynamic noun generalization: Moment-to-moment interactions shape children's naming biases, Infancy 11 (1) (2007), pp. 97-110.

Samuelson and Horst, 20084 L. K. Samuelson and J. S. Horst, Confronting complexity: Insights from the details of behavior over multiple timescales, Developmental Science 11 (2008), pp. 209-215.

Samuelson et al., 2008 L. K. Samuelson, J. S. Horst, A. R. Schutte, and B. N. Dobbertin, Rigid thinking about deformables: Do children sometimes overgeneralize the shape bias, Journal of Child Language 35 (2008), pp. 559-589.

Samuelson and Smith, 19994 L. K. Samuelson and L. B. Smith, Early noun vocabularies: Do ontology, category organization and syntax correspond?, Cognition 73 (1) (1999), pp. 1-33.

Samuelson and Smith, 2000a L. K. Samuelson and L. B. Smith, Children's attention to rigid and deformable shape in naming and non-naming tasks, Child Development 71 (6) (2000), pp. 1555-1570.

Samuelson and Smith, 2000b 4 L. K. Samuelson and L. B. Smith, Grounding development in cognitive processes, Child Development 71 (2000), pp. 98-106.

Sandhofer, 2001 C. Sandhofer, Structure in parents' input: Effects of categorization versus comparison. In: A. H. Do, L. Dominguez and A. Johansen, Editors, Proceedings of the 25th annual Boston University conference on language development Vol. 2, Cascadilla Press, Sommerville, MA (2001), pp. 657-667.

Schöner and Dineva, 2007 G. Schöner and E. Dineva, Dynamic instabilities as mechanisms for emergence, Developmental Science $\mathbf{1 0}$ (2007), pp. 69-74.

Schutte and Spencer, in press 4 A. R. Schutte and J. P. Spencer, Tests of the dynamic field theory and the spatial precision hypothesis: A developmental transition in spatial working memory. Journal of Experimental Psychology: Human Perception and Performance (in press).

Schutte et al., 2003 A. R. Schutte, J. P. Spencer, and G. Schöner, Testing the dynamic field theory: Working memory for locations becomes more spatially precise over development, Child Development 74 (5) (2003), pp. 1393-1417.

Simmering et al., $2008 \leftarrow$ V. R. Simmering, A. R. Schutte, and J. P. Spencer, Generalizing the dynamic field theory of spatial working memory across real and developmental time scales. In S. Becker (Ed.), Computational cognitive neuroscience. Brain Research, 1202, 6886 (special issue; 2008).

Simmering et al., $2006-$ V. R. Simmering, J. P. Spencer, and G. Schöner, Reference-related inhibition produces enhanced position discrimination and fast repulsion near axes of symmetry, Perception and Psychophysics 63 (2006), pp. 1027-1046.
Skarda and Freeman, 1987 C. A. Skarda and W. J. Freeman, How brains make chaos in order to make sense of the world, Behavioral and Brain Sciences 10 (2) (1987), pp. 161-195.

Smith, 20004 L. B. Smith, Learning how to learn words: an associative crane. In: R. M. Golinkoff and K. Hersh-Pasek, Editors, Becoming a word learner. A debate on lexical acquisition, Oxford University Press, New York (2000), pp. 51-80.

Smith, 20014 L. B. Smith, How domain-general processes my create domain-specific biases. In: M. Bowerman and S. Levinson, Editors, Language acquisition and conceptual development, Cambridge University Press, Cambridge, MA (2001), pp. 101-131.

Smith et al., 19924 L. B. Smith, S. S. Jones, and B. Landau, Count nouns, adjectives, and perceptual properties in children's novel word interpretations, Developmental Psychology 28 (2) (1992), pp. 273-286.

Smith et al., 20024 L. B. Smith, S. S. Jones, B. Landau, L. GershkoffStowe, and L. K. Samuelson, Object name learning provides onthe-job training for attention, Psychological Science 13 (1) (2002), pp. 13-19.

Smith et al., 2003 L. B. Smith, S. Jones, H. Yoshida, and E. Colunga, Whose DAM account? Attentional learning explains Booth and Waxman, Cognition 87 (2003), pp. 209-213.

Smith et al., 1999 L. B. Smith, E. Thelen, R. Titzer, and D. McLin, Knowing in the context of acting: The task dynamics of the A-notB error, Psychological Review 106 (2) (1999), pp. 235-260.

Smith and Samuelson, 20064 L. B. Smith and L. K. Samuelson, An attentional learning account of the shape bias: Reply to Cimpian \& Markman (2005) and Booth, Waxman \& Huang (2005), Developmental Psychology 42 (6) (2006), pp. 1339-1343.

Sophian, $1997 \varangle$ C. Sophian, Beyond competence: The significance of performance of conceptual development, Cognitive Development 12 (1997), pp. 281-303.

Spelke et al., 1992 E. S. Spelke, K. Breinlinger, J. Macomber, and K. Jacobson, Origins of knowledge, Psychological Review 99 (4) (1992), pp. 605-632.

Spencer et al., 2007 J. P. Spencer, V. R. Simmering, A. R. Schutte, and G. Schöner, What does theoretical neuroscience have to offer the study of behavioral development? Insights from a dynamic field theory of spatial cognition. In: J. M. Plumert and J. P. Spencer, Editors, The Emerging Spatial Mind, Oxford University Press, Oxford (2007), pp. 321-361.

Spencer et al., in press 4 J. P. Spencer, E. Dineva, and G. Schöner, Moving toward a grand theory while valuing the importance of the initial conditions. In J. P. Spencer, M. S. Thomas, \& J. L. McClelland (Eds.), Toward a unified theory of development: Connectionism and dynamic systems theory re-considered. New York: Oxford University Press (in press).

Spencer and Schöner, 2003 J. P. Spencer and G. Schöner, Bridging the representational gap in the dynamic systems approach to development, Developmental Science 6 (2003), pp. 392-412.

Spencer et al., 20064 J. P. Spencer, V. R. Simmering, and A. R. Schutte, Towards a formal theory of flexible spatial behavior: Geometric category biases generalize across pointing and verbal response types, Journal of Experimental Psychology: Human Perception and Performance 32 (2) (2006), pp. 473-490.

Spivey and Dale, $2006 \checkmark$ M. J. Spivey and R. Dale, Continuous dynamics in real-time cognition, Current Directions in Psychological Science 15 (2006), pp. 207-211.

Starkey et al., 1990 P. Starkey, E. S. Spelke, and R. Gelman, Numerical abstraction by human infants, Cognition 36 (1990), pp. 97-128.

Thelen et al., 20014 E. Thelen, G. Schöner, C. Scheier, and L. B. Smith, The dynamics of embodiment: A field theory of infant perseverative reaching, Behavioural and Brain Sciences 24 (2001), pp. $1-86$.

Thelen and Smith, 1994 E. Thelen and L. B. Smith, A dynamic systems approach to the development of cognition and action, MIT Press, Cambridge, MA (1994).

Thelen and Smith, 1997 E. Thelen and L. B. Smith, Dynamic systems theories (5th ed.). In: D. Khun and R. Seigler, Editors, Theoretical models of human development. Volume 1 of the Handbook of child psychology Vol. 1, Wiley, New York (1997), pp. 563-634.

Yoshida and Smith, 2003 H. Yoshida and L. B. Smith, Shifting ontological boundaries: How Japanese- and English-speaking children generalize names for animals and artifacts, Developmental Science 6 (1) (2003), pp. 1-17. 